\title{
Der free flow of information im Wandel des digitalen Zeitalters: Eine Bestandsaufnahme der internetbezogenen Rechtsprechung des EGMR zu Art. 10 EMRK
}

\author{
Tobias Brings-Wiesen, Mag. iur./Alicia Damberg-Jänsch, LL.M.*
}

I. Einleitung

II. Spezifika in der Rechtspre-

chung des EGMR

1. Welcher Stellenwert und welche Wirkkraft werden dem Internet als Informations- und Kommunikationsmedium zugesprochen?

a) Beschränkungen der Internetnutzung

aa) Restriktionen zulasten der allgemeinen Internetnutzer

(1) Nicht jeder ist immer ein „Opfer"

(2) Rechtfertigungsanforderungen an staatliche Sperrund Löschverfügungen

bb) Gleiches Recht für alle? Restriktionen der Internetnutzung im Strafvollzug 296

b) Die Wirkkraft im Internet 299
2. Wie sind Rolle, Funktion und Arbeitsweise von (neuen) Akteuren im digitalen Informations- und Kommunikationsprozess einzuordnen? 301

a) Das Internet und die journalistische Arbeit ... 301

aa) Die Trennung von Privatperson und Journalist ........... 302

bb) Die neuen ,watchdogs“" ............... 303

cc) Online-Archive ..... 306

b) Intermediäre ............ 308

aa) Öffentliche Kommunikationsräume im Internet ............. 309

bb) Suchmaschinen ..... 315

3. Welche Entwicklungen zeigen sich angesichts eines sich wandelnden Kommunikationsmodus? 316

a) Internetspezifische Formen der Kommunikation 316

b) „Der gute Ton“" im Internet $\ldots \ldots \ldots \ldots \ldots \ldots \ldots . . . \ldots . \ldots 318$

III. Fazit 319

* Tobias Brings-Wiesen ist Doktorand am Institut für Medienrecht und Kommunikationsrecht der Universität zu Köln, Lehrstuhl für Öffentliches Recht und Medienrecht Professor Dr. Karl-E. Hain (E-Mail: tobias.brings-wiesen@uni-koeln.de),Alicia Damberg-Jänsch ist Volljuristin im Justitiariat der Deutschen Welle (E-Mail: alicia.damberg-jaensch@dw.com). Alle zitierten Internetquellen wurden zuletzt abgerufen am 7.6.2020; einzelne besonders relevante Judikate des EGMR konnten noch bis Anfang Juli 2020 berücksichtigt werden. - Der Beitrag ist inspiriert von den Inhalten der von der Deutschen Welle und dem Institut für Medienrecht und Kommunikationsrecht der Universität zu Köln organisierten Veranstaltungsreihe ZOOM - Internationales Medienrecht (s. dazu: https://dw.com/medienrecht). Die Autoren danken den Reviewern für ihre Impulse zur Optimierung des Textes. 


\begin{abstract}
Die signifikanten Veränderungen im menschlichen Kommunikations- und Informationsprozess durch das Internet haben in den vergangenen Jahren graduell Ausdruck in Recht und Rechtsprechung gefunden. Der folgende Beitrag analysiert, wie der Europäische Gerichtshof für Menschenrechte seine über Jahrzehnte etablierte Rechtsprechung zu Art. 10 der Europäischen Menschenrechtskonvention unter klar zum Ausdruck kommender Berücksichtigung der Spezifika des neuen Mediums bestätigt und behutsam fortentwickelt hat sowie zu welchen Themen angesichts der „Communicated Cases" in (naher) Zukunft Judikate zu erwarten sind. Dabei werden drei Analysefelder fokussiert: Stellenwert und Wirkkraft des Mediums an sich - Rolle, Funktion und Arbeitsweise von (neuen) Akteuren im digitalen Kommunikations- und Informationsprozess - Entwicklungen des Kommunikationsmodus. In Anerkennung der Wechselwirkungen innerhalb des europäischen Grundrechtsverbunds werden darüber hinaus Querbezüge zu verwandten Diskussionen auf nationaler und EU-Ebene hergestellt.
\end{abstract}

\title{
I. Einleitung
}

Die Idee eines ,free flow of information" ist nicht bloß Grundprinzip der transnationalen Kommunikationsordnung, ${ }^{1}$ sondern auch gedankliches Fundament von Art. 10 der Europäischen Menschenrechtskonvention (EMRK) ${ }^{2}$. Dessen Anliegen ist es, den Kommunikations- und Informationsprozess im Interesse der Selbstverwirklichung des Einzelnen und der demokratischen Gesamtgesellschaft frei von jedweder Form der ungerechtfertigten Behinderung zu gewährleisten. ${ }^{3}$

Aufgrund seiner Kapazitäten und Nutzungsmöglichkeiten hat das Internet diesen Prozess in den vergangenen Jahren signifikant beeinflusst. Menschliche Kommunikation und

1 S. dazu nur Wein, UFITA 105 (1987), 137 ff.; auch Recommendation CM/Rec(2015)6 of the Committee of Ministers to member States on the free, transboundary flow of information on the Internet, abrufbar unter: https://go.coe.int/65OUz.

2 Ausdrückliche Erwähnung des Terminus in EGMR, 5.11.2002 - 38743/97, Rn. 40 - Demuth v. Switzerland; 9.6.2009 - 17095/03, Rn. 33 - Cihan Öztürk v. Turkey; 14.6.2011 - 28040/08, Rn. 48 - Aquilina and Others v. Malta; 21.2.2012 - 32131/08 und 41617/08, Rn. 50 - Tuşalp v. Turkey; ähnlich auch EGMR (GK), 16.6.2015 - 64569/09, Rn. 147 - Delfi AS v. Estonia = NJW 2015, 2863; 24.4.2018 - 62357/14, Rn. 105 - Benedik v. Slovenia. - Alle Entscheidungen werden zitiert nach der Online-Entscheidungsdatenbank des EGMR, erreichbar unter: https://hudoc.echr.coe.int/. Verbindlich sind nur die Veröffentlichungen des Gerichtshofs in französischer und englischer Sprache. Die deutschen Übersetzungen und Paraphrasierungen der Judikate wurden von den Autoren persönlich vorgenommen. Hinweise auf deutsche Übersetzungen in Fachzeitschriften erfolgen als ergänzender Service. Zusammenfassungen zahlreicher behandelter Entscheidungen finden sich bei Voorhoof et al., Freedom of expression, the Media and Journalists, 5th Ed. 2020.

3 Vgl. zu diesem dualistischen Schutzansatz die st. Rspr. des EGMR bereits 7.12.1976 - 5493/72, Rn. 49 - Handyside v. UK; aus jüngerer Zeit 13.2.2020 - 63571/16 u.a., Rn. 164 - Ibrahimov and Mammadov v. Azerbaijan. Für eine theoretische Fundierung Oster, Kommunikationsdeliktsrecht, 2019, S. $133 \mathrm{ff}$. 
Information findet heute zu einem nicht unerheblichen Anteil online statt. Dabei sind aus bloßen Rezipienten ${ }^{4}$ Nutzer, aus einfacher Konfrontation ist Interaktion geworden. Das Internet hat die tatsächlichen Rahmenbedingungen des Informationsflusses derart fundamental verändert, dass die Frage nach notwendigen korrespondierenden Modifikationen der rechtlichen Rahmenbedingungen zur ständigen Aufgabe auch der medienrechtlichen Wissenschaft und Praxis avanciert ist. Der jahrzehntelang etablierte Bewertungsmaßstab für Kommunikanten und Kommunikate ist im Wandel.

Der Europäische Gerichtshof für Menschenrechte (EGMR) agiert bei der erforderlichen Würdigung dieses Wandels im europäischen Grundrechtsgefüge als „key player". Seine Rechtsprechung hat nicht bloß Auswirkungen auf die Auslegung der Grundrechte der Europäischen Union (vgl. Art. 52 Abs. 3 und Art. 53 GRCh), ${ }^{5}$ sondern auch auf die der Grundrechtsordnungen der Konventionsstaaten. Im deutschen Recht dienen die Judikate des EGMR bekanntermaßen als „Auslegungshilfen“, die - selbst fernab einer unmittelbaren Verbindlichkeit gemäß Art. 46 Abs. 1 EMRK - eine kontextsensible Leit- und Orientierungswirkung für die Interpretation des Grundgesetzes entfalten ${ }^{6}{ }^{7}$

Während das Internet in der Rechtsprechung der EGMR zunächst noch eine untergeordnete Rolle spielte, ${ }^{8}$ hat sich der Wandel der Medienlandschaft schrittweise in den Entscheidungen abgebildet. Mit einer gewissen Verzögerung, die der obligatorischen Erschöpfung des innerstaatlichen Rechtswegs (Art. 35 Abs. 1 EMRK) und der langen Verfahrensdauer vor dem EGMR geschuldet sein dürfte, hat sich der Gerichtshof bereits einer ganzen Reihe von Problemkomplexen zuwenden können, die die Fachdiskussionen auf deutscher und EU-Ebene schon seit Längerem bestimmt hatten.

4 Zur einfacheren und inklusionsfreundlichen Lesbarkeit verwendet dieser Aufsatz das generische Maskulinum. Aussagen zu geschlechtlicher Identität sind damit nicht verbunden und auch nicht beabsichtigt.

5 S. zu Art. 52 Abs. 3 GRCh nur Cornils, in: Grabenwarter, § 5, Rn. 27 ff.; zu Art. 53 GRCh nur Krä$m e r$, in: Stern/Sachs, § 53, passim; s. konkret zu Art. 11 GRCh aus jüngerer Zeit EuGH, 14.2.2019 C-345/17, K\&R 2019, 252 (Rn. 65 f.) - Buivids; 29.7.2019 - C-516/17, K\&R 2019, 574 (Rn. 57 f.) - Spiegel Online; 29.7.2019 - C-469/17, WRP 2019, 1170 (Rn. 73 f.) - Funke Medien NRW; s. auch EuGH, 24.9.2019 - C-136/17, K\&R 2019, 710 (Rn. 76) - GC u.a.; zu Art. 11 GRCh im Überblick Brings-Wiesen, in: Spindler/Schuster, Erster Teil. B. Rn. 13 ff. (insb. zur Entsprechung gemäß Art. 52 Abs. 3 GRCh Rn. 14 f.).

6 Dazu näher bereits Brings-Wiesen, in: Spindler/Schuster, Erster Teil. A. Rn. 9 ff. S. zul. insb. BVerfG, 12.6.2018 - 2 BvR 1738/12 u.a., BVerfGE 148, 296 (Rn. 126 ff.); zu den Besonderheiten dieses Urteils Haug, NJW 2018, 2674 ff.; abstrahierend Lepsius, JZ 2019, 793 ff. S. zur völkerrechtsfreundlichen Praxis des BVerfG jüngst auch Voßkuhle, ZaöRV 79 (2019), 481 (483ff.).

7 S. zu Zusammenspiel und Wechselwirkung der Grundrechtsebenen aus jüngerer Zeit insb. BVerfG, 6.11.2019 - 1 BvR 16/13, K\&R 2020, 51 (Rn. 45 ff.) - Recht auf Vergessen I; 6.11.2019 - 1 BvR 276/17, K\&R 2020, 59, Rn. 44, 59 - Recht auf Vergessen II.

8 Heißl, EGMR und Internet, 2012, S. 61 (64). 
Der folgende Beitrag soll ein Schlaglicht darauf werfen, welchen klar erkennbaren Einfluss die Funktionsmechanismen des Internets auf die materiellen Maßstäbe ${ }^{9}$ des EGMR in der Auslegung von Art. 10 EMRK bis heute bereits ${ }^{10}$ genommen haben und welche Entwicklungstendenzen sich abzeichnen. Dabei sollen an den entsprechenden Stellen auch Parallelen zur Judikatur der deutschen und der Unionsgerichte aufgezeigt werden.

\section{Spezifika in der Rechtsprechung des EGMR}

Getreu dem Mantra „what applies offline, also applies online“ hält der Gerichtshof auch bei der Bewertung internetbezogener Sachverhalte am Maßstab von Art. 10 EMRK an seinem bekannten Prüfungsschema fest. Er begutachtet, ob ein Eingriff (,interference“) in den Schutzbereich (,applicability“, „,ambit") vorliegt und ob dieser gerechtfertigt werden kann, wofür er auf einer hinreichenden rechtlichen Grundlage (Gesetzesvorbehalt, ,prescribed by law") beruhen, einen legitimen Zweck (,legitimate aim") verfolgen und die Anforderungen des Verhältnismäßigkeitsprinzips (im weiteren Sinne, „necessary in a democratic society“) wahren muss. ${ }^{11}$

Dabei ist der EGMR bislang nicht zu grundlegenden Modifikationen gezwungen gewesen. Ganz im Sinne der „living instrument"-Doktrin ${ }^{12}$ hat der Gerichtshof seine Rechtsprechung unter Berücksichtigung der Spezifika internetbezogener Sachverhalte auf fast allen Prüfungsebenen lediglich problemorientiert fortentwickelt. Die folgende Bestandsaufnahme möchte diese Fortentwicklung hinsichtlich dreier zentraler Fragenkomplexe nachvollziehen: (1.) Welcher Stellenwert und welche Wirkkraft werden dem Internet als Kommunikations- und Informationsmedium zugesprochen? (2.) Wie sind Rolle, Funktion und Arbeits-

9 Fragen der Zuständigkeit des EGMR bzw. des Geltungsbereichs der EMRK in internetbezogenen Sachverhalten - s. dazu Research Division des EGMR, Internet: case-law of the European Court of Human Rights, Juni 2015, S. 4 ff. - sowie die Wirkungen auf die Bewertungsmaßstäbe innerhalb anderer Grundrechte der EMRK - s. insb. zu Art. 8 EMRK Paefgen, Persönlichkeitsrechte im Internet, 2017, passim - sollen nicht thematisiert werden.

10 S. zu diesem dynamischen Prozess in der Vergangenheit bereits Uerpmann-Wittzack/JankowskaGilberg, MMR 2008, 83 ff.; Heißl, EGMR und Internet, 2012, S. 61 ff.; Vajić/Panayotis, The Internet and Freedom of Expression, 2012, S. 391 ff.; Benedek/Kettemann, Freedom of expression and the Internet, 2013, passim; Barata Mir/Bassini, Freedom of expression in the Internet, 2016, S. $71 \mathrm{ff}$; Buffa, Freedom of Expression in the Internet Society, 2016, passim; O'Leary, Irish Jurist 2018, 59 ff. (insb. 69 ff.); Kettemann/Benedek, Freedom of expression online, 2019, S. 58 ff.; jüngst Voorhoof, Same standards, different tools?, 2020, S. $11 \mathrm{ff}$. - S. auch die verschiedenen offiziellen Materialien zu diesem Themenkomplex: Research Division des EGMR, Internet: case-law of the European Court of Human Rights, Juni 2015; Press Unit des EGMR, Fact Sheet zu „Access to Internet and freedom to receive and impart information and ideas", Juni 2020; auch Research and Library Division des EGMR, Article 10: Expression and advertising of political positions through the media/Internet in the context of elections/referendums, 22.8.2018; Press Unit des EGMR, Fact Sheet zu „New technologies“, Februar 2020.

11 Zum Aufbau der Prüfung bereits Brings-Wiesen, in: Spindler/Schuster, Erster Teil. A. Rn. 14 ff.

12 S. dazu ausführlich Letsas, The ECHR as a living instrument, 2013, S. $106 \mathrm{ff}$. 
weise von (neuen) Akteuren im digitalen Kommunikations- und Informationsprozess einzuordnen? (3.) Welche Entwicklungen zeigen sich angesichts eines sich wandelnden Kommunikationsmodus?

Ausgangspunkt der Bestandsaufnahme ist der einheitliche und weite Schutzbereich von Art. 10 EMRK. Diesem unterfällt seit jeher der gesamte Informations- und Kommunikationsprozess, ${ }^{13}$ unabhängig davon, auf welchem Wege er vollzogen wird. Daher ist es konsistent, dass der EGMR auch die Verbreitung ${ }^{14}$ und die Rezeption ${ }^{15}$ von Kommunikaten und Informationen jeder Art über das Internet eben diesem Schutzbereich zuordnet. Die ständige Rechtsprechung ${ }^{16}$ des Gerichtshofs offenbart eine derartige Offenheit indes ebenfalls für die Bereitstellung der Kommunikations- und Informationsmittel (,means of disseminati-

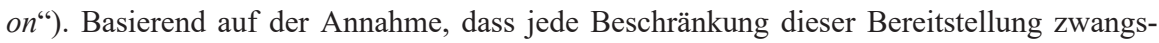
läufig das Recht auf Empfang und Weitergabe von Informationen beeinträchtige, hat der EGMR in jüngerer Zeit die Bereitstellung diverser Formen von Internetdiensten dem Schutz von Art. 10 EMRK unterstellt: den Webhosting-Dienst „Google Sites“"17, die FileSharing-Website „The Pirate Bay“"18, die Video-Sharing-Plattform „YouTube"19 sowie jüngst die Mobile App „Cast an invalid ballot“ einer ungarischen Partei ${ }^{20}$. Auch die Bereit-

13 Brings-Wiesen, in: Spindler/Schuster, Erster Teil. A. Rn. 17.

14 S. nur EGMR, 18.12.2012 - 3111/10, Rn. 48 f. - Ahmet Yildirim v. Turkey: Betreiben einer eigenen Google Site; 17.7.2018 - 38004/12, Rn. 251 - Mariya Alekhina and Others v. Russia: Verbreiten eines Videos über eine eigene Website; 30.4.2019 - 48310/16 und 59663/17, Rn. 84 - Kablis v. Russia: Betreiben eines VKontakte-Accounts und Posten von Blogeinträgen; s. zul. auch zur Beschlagnahme eines Servers mit Informationen zur Bereitstellung einer Website EGMR, 26.3.2020 - 44229/11, Rn. 59 - Pendov v. Bulgaria.

15 S. nur EGMR, 1.12.2015 - 48226/10 und 14027/11, Rn. 50 - Cengiz and Others v. Turkey = NJOZ 2017, 1214: Zugriff auf YouTube; 19.1.2016 - 17429/10, Rn. 43 - Kalda v. Estonia $=$ NJOZ 2018, 1598, und 17.1.2017 - 21575/08, Rn. 53 - Jankovskis v. Lithuania = NJOZ 2018, 1158: Zugang zu bestimmten Websites im Strafvollzug.

16 S. nur betr. den Eigentümer einer Zeitung EGMR (GK), 8.7.1999 - 26682/95, Rn. 43, 63 - Sürek v. Turkey (no. 1); den Verleger eines Buches EGMR (GK), 28.9.1999 - 22479/94, Rn. 49 - Öztürk v. Turkey; die Verantwortliche für einen Flyer EGMR, 14.10.2010 - 4260/04, Rn. 42 - Andrushko v. Russia.

17 EGMR (GK), 20.1.2020 - 201/17, Rn. 87 - Magyar Kétfarkú Kutya Párt v. Hungary, unter Rekurs auf EGMR, 18.12.2012 - 3111/10, Rn. 49 - Ahmet Yildirim v. Turkey. Vgl. auch zu „Blogger.com“EGMR, 19.9.2017 - 3877/14, Rn. 79, 90 - Tamiz v. UK.

18 EGMR, 19.2.2013 - 40397/12 - Neij and Sunde Kolmisoppi v. Sweden.

19 EGMR, 1.12.2015 - 48226/10 und 14027/11, Rn. 52, 56 - Cengiz and Others v. Turkey = NJOZ 2017, 1214.

20 EGMR, 13.1.2019 - 201/17, Rn. 37 - Magyar Kétfarkú Kutya Párt v. Hungary; deutlicher noch EGMR (GK), 20.1.2020 - 201/17, Rn. 87 f., 91 - Magyar Kétfarkú Kutya Párt v. Hungary: ,providing a forum for third-party content". - Darüber hinaus wurde in der besonderen Zweckrichtung der besagten App, der Dokumentation der Kritik von Wählern, auch ein eigenständiger Aussagegehalt erkannt, a.aen.Oen., Rn. 37 bzw. Rn. 89 ff. 
stellung von in Diensten integrierten Diskussions- und Kommentarbereichen unterfällt dem Schutzbereich. ${ }^{21}$

\section{Welcher Stellenwert und welche Wirkkraft werden dem Internet als Informations- und Kommunikationsmedium zugesprochen?}

Bei einer Bestandsaufnahme der internetbezogenen Rechtsprechung zu Art. 10 EMRK lohnt zunächst ein Blick auf die wiederkehrenden Feststellungen des EGMR zur Relevanz und den zentralen Charakteristika des Mediums an sich. Diese bisweilen ausdrücklich als „allgemeine Prinzipien“ (,general principles") ausgewiesenen Passagen bilden in der primär einzelfallorientierten Judikatur des Gerichtshofs feste Pfeiler des Koordinatensystems seiner Prüfung.

Kaum überraschen dürfte es, dass der EGMR die Bedeutung des Internets für den alltäglichen Kommunikations- und Informationsprozess als hoch einschätzt: Angesichts seiner Zugänglichkeit sowie seiner enormen Speicher- und Übertragungskapazität spiele das Internet eine wichtige Rolle dabei, den Zugang der Allgemeinheit zu Nachrichten zu fördern und die Verbreitung von Informationen allgemein zu erleichtern. ${ }^{22}$ Es sei gar zu einem der wichtigsten Mittel geworden, mit denen Individuen ihr Recht auf Äußerungs- und Informationsfreiheit ausübten, da es wesentliche Instrumente zur Teilnahme an Aktivitäten und Diskussionen betreffend Anliegen von politischem oder allgemeinem Interesse biete. ${ }^{23}$ Insbesondere hinsichtlich der Möglichkeiten zur Verbreitung nutzergenerierter Inhalte biete es eine bislang beispiellose Plattform. ${ }^{24}$ Auch sei eine zunehmende Menge an Diensten und Informationen nur noch im Internet verfügbar. ${ }^{25}$ In diesem Bewusstsein verglich der EGMR

21 EGMR (GK), 16.6.2015 - 64569/09, Rn. 118 - Delfi AS v. Estonia = NJW 2015, 2863; EGMR, 2.2.2016 - 22947/13, Rn. 45 - Magyar Tartalomszolgáltatók Egyesülete and Index.hu Zrt v. Hungary $=$ NJW 2017, 2091; 7.2.2017 - 74742/14, Rn. 29 - Pihl v. Sweden; 19.3.2019 - 43624/14, Rn. 68 - Høiness v. Norway.

22 EGMR, 10.3.2009 - 3002/03 und 23676/03, Rn. 27 - Times Newspapers Ltd (Nos. 1 and 2) v. UK; erstmalig als ,general principle“ genannt in EGMR (GK), 16.6.2015 - 64569/09, Rn. 133 - Delfi AS v. Estonia = NJW 2015, 2863; zul. EGMR, 30.4.2019-48310/16 und 59663/17, Rn. 81 - Kablis v. Russia. S. zur Bedeutung des Internets auch Recommendation CM/Rec(2014)6 of the Committee of Ministers to member States on a Guide to human rights for Internet users and explanatory, abrufbar unter: https://rm.coe.int/16804d5b31; Recommendation CM/Rec(2016)5 of the Committee of Ministers to member States on Internet freedom, abrufbar unter: https://go.coe.int/fSGw A.

23 EGMR, 18.12.2012 - 3111/10, Rn. 54 - Ahmet Yildirim v. Turkey; 1.12.2015 - 48226/10 und 14027/11, Rn. 49 - Cengiz and Others v. Turkey = NJOZ 2017, 1214.

24 EGMR (GK), 16.6.2015 - 64569/09, Rn. 110 - Delfi AS v. Estonia = NJW 2015, 2863; aufgegriffen in EGMR, 1.12.2015 - 48226/10 und 14027/11, Rn. 52 - Cengiz and Others v. Turkey = NJOZ 2017, 1214; erstmalig als ,general principle“ genannt in EGMR, 30.4.2019 - 48310/16 und 59663/17, Rn. 81 - Kablis v. Russia.

25 EGMR, 19.1.2016 - 17429/10, Rn. 52 - Kalda v. Estonia = NJOZ 2018, 1598; 17.1.2017 21575/08, Rn. 62 - Jankovskis v. Lithuania = NJOZ 2018, 1158. 
jüngst die Sperrung des Zugriffs auf eine gesamte Website als „extreme Maßnahme“ mit dem Verbot einer Zeitung oder eines Fernsehsenders. ${ }^{26}$

Die Bedeutung des Internets beruht zweifellos auf seinen zentralen Charakteristika, deren Janusköpfigkeit jedoch die Bewertung eines Einzelfalls nicht unerheblich komplizieren kann: Denn die Möglichkeit der weltweiten, sekundenschnellen Verbreitung von Informationen $^{27}$ (in völliger Anonymität ${ }^{28}$ ) und deren dauerhafte Verfügbarkeit ${ }^{29}$ kann zugleich Segen und Fluch für den ,free flow of information" sowie andere individuelle und kollektive Rechtsgüter sein. ${ }^{30}$ Der EGMR sieht insbesondere das Recht auf Achtung des Privatlebens gemäß Art. 8 EMRK einem erhöhten Risiko ausgesetzt und verlangt - unter Berücksichtigung der Gleichwertigkeit der konfligierenden Interessen - entsprechend effektive Rechtsschutzmechanismen. ${ }^{31}$

\section{a) Beschränkungen der Internetnutzung}

In Anbetracht der Bedeutung des Mediums für den heutigen Kommunikations- und Informationsprozess rückt eine Frage besonders in den Fokus: Unter welchen Bedingungen kann die Internetnutzung in zulässiger Weise beschränkt werden? Zwar ist die einschlägige Rechtsprechung des EGMR bislang überschaubar, gleichwohl sind erste Leitlinien und Ent-

26 EGMR, 23.6.2020 - 20159/15, Rn. 34 - Bulgakov v. Russia; 23.6.2020 - 12468/15, 23489/15 und 19074/16, Rn. 37 - OOO Flavus and Others v. Russia; 23.6.2020 - 10795/14, Rn. 38 Vladimir Kharitonov v. Russia; jeweils unter Rekurs auf Ziff. 3 lit. a. der Joint declaration on freedom of expression and the Internet by the UN Special Rapporteur on Freedom of Opinion and Expression, OSCE Representative on Freedom of the Media, OAS Special Rapporteur on Freedom of Expression and ACHPR Special Rapporteur on Freedom of Expression and Access to Information on 1 June 2011, abrufbar unter: https://www.osce.org/fom/78309.

27 S. zur diesbezüglichen Ambivalenz EGMR (GK), 16.6.2015 - 64569/09, Rn. 110 - Delfi AS v. Estonia $=$ NJW 2015, 2863; s. auch EGMR, 26.11.2015 - 3690/10, Rn. 67, 72 - Annen v. Germany = NJW 2016, 1867; vgl. zur Verstärkung von Reichweite im Internet bereits EGMR, 16.7.2009 10883/05, Rn. 36 - Willem c. France; tendenziell auch EGMR, 25.10.2018 - 38450/12, Rn. 51 E.S. v. Austria.

28 S. zur diesbezüglichen Ambivalenz und den Abstufungen von Anonymität EGMR (GK), 16.6.2015 - 64569/09, Rn. 147 ff. - Delfi AS v. Estonia = NJW 2015, 2863; vgl. auch EGMR, 2.12.2008 - 2872/02, Rn. 34, 37 f., 48 - K.U. v. Finland; 24.11.2015 - 72966/13, Rn. 39 f. - Kucharczyk v. Poland; 24.4.2018 - 62357/14, Rn. 105 f., 117 - Benedik v. Slovenia; zur Abgrenzung von Art. 8 EMRK s. EGMR, 30.1.2020 - 50001/12, Rn. 60, 62 - Breyer v. Germany = NLMR 2020, 32. Zur Frage eines Rechts auf Anonymität nach dem GG aus jüngerer Zeit Kersten, JuS 2017, $193 \mathrm{ff}$.

29 S. noch speziell zur Rspr. betr. Online-Archive unter II. 2. a) cc).

30 EGMR, 5.5.2011 - 33014/05, Rn. 63 - Editorial Board of Pravoye and Shtekel v. Ukraine; erstmalig als ,general principle“ genannt in EGMR (GK), 16.6.2015 - 64569/09, Rn. 133 - Delfi AS v. Estonia $=$ NJW 2015, 2863; 4.12.2018 - 11257/16, Rn. 66 - Magyar Jeti Zrt v. Hungary = NJW 2019, 3201.

31 EGMR (GK), 16.6.2015 - 64569/09, Rn. 110 - Delfi AS v. Estonia = NJW 2015, 2863; 30.1.2018 - 59040/09, Rn. 28 - Oktar c. Turquie. S. zum im Vergleich zur Presse erhöhten Risiko auch bereits EGMR, 5.5.2011 - 33014/05, Rn. 63 - Editorial Board of Pravoye and Shtekel v. Ukraine. 
wicklungstendenzen erkennbar. Bei deren Analyse ist situativ grundlegend zwischen Restriktionen zulasten von Internetnutzern allgemein und zulasten der Sondergruppe der Inhaftierten zu unterscheiden. ${ }^{32}$

\section{aa) Restriktionen zulasten der allgemeinen Internetnutzer}

Die denkbaren Fallkonstellationen staatlicher Eingriffe in den Kommunikations- und Informationsprozess sind auch im Internet so vielgestaltig wie die Ausprägungen des Schutzbereichs von Art. 10 EMRK. ${ }^{33}$ Sie reichen von gesetzlichen Ge- und Verboten über zivil- und strafgerichtliche Urteile bis hin zu behördlichen Maßnahmen. ${ }^{34}$ Klare Konfliktlinien offenbaren sich im Falle von Sperr- bzw. Löschverfügungen, mit denen der Staat selbst ${ }^{35}$ gezielt den Zugriff auf bestimmte Dienste und Inhalte ${ }^{36}$ verhindert.

\section{(1) Nicht jeder ist immer ein „Opfer“}

Insofern ist zunächst zu berücksichtigen, dass sich die verschiedenen Formen der Beschränkung des Kommunikations- und Informationsprozesses im Internet auf dessen Teilnehmer unterschiedlich intensiv auswirken können. Es ist daher im Einzelfall zu klären, ob eine Rechtsverletzung bereits mangels eines erheblichen Eingriffs ${ }^{37}$ von vornherein ausgeschlossen ist. Denn bei Negation des Opferstatus („victim status“) kann eine Individualbeschwerde gemäß Art. 34 Satz 1 EMRK gar als unzulässig abgewiesen werden. Diese grundsätzliche Problematik ${ }^{38}$ spitzt sich im Falle von Sperr- und Löschverfügungen im Internet auf die Frage zu, wie weit der Kreis der Opfer überhaupt zu ziehen ist. Dabei ist die Konfliktlage im Rahmen von Art. 10 EMRK nicht völlig neu: Bereits in der Vergangenheit verneinte der EGMR im Falle staatlicher Beschränkungen von allgemein zugänglichen Informationsquel-

32 S. dazu auch Press Unit des EGMR, Factsheet zu „Access to Internet and freedom to receive and impart information and ideas", Juni 2020.

33 S. zur „Spiegelbildlichkeit“ der beiden Prüfungsebenen bereits Brings-Wiesen, in: Spindler/Schuster, Erster Teil. A. Rn. 31.

34 Im Einzelfall kann auch staatliches Unterlassen gegen eine Schutzpflicht (,positive obligation“) nach Art. 10 EMRK verstoßen, s. dazu Brings-Wiesen, in: Spindler/Schuster, Erster Teil. A. Rn. 33.

35 Davon abzugrenzen sind die abstrakteren Vorgaben an Anbieter von Online-Archiven und Intermediäre, s. dazu unter II. 2. a) cc) und b).

36 Modifikationen der Prüfungsmaßstäbe betreffend bestimmte Akteure und den Inhalt der Kommunikation online sollen gesondert behandelt werden, s. dazu unter II. 2. a) und 3.

37 Für einen Überblick über die Variabilität möglicher Eingriffe in Art. 10 EMRK s. EGMR, 12.11.2019 - 68995/13, Rn. 71 - Schweizerische Radio- und Fernsehgesellschaft et autres c. Suisse.

38 S. dazu nur Schäfer, in: Karpenstein/Mayer, Art. 34 Rn. 61 ff. Die Voraussetzung erfüllt auch eine Filterfunktion mit dem Ziel des Schutzes der Funktionsfähigkeit des Gerichtshofs, s. zum Ziel der Zulässigkeitsvoraussetzungen Schäfer, in: Karpenstein/Mayer, Art. 35 Rn. 2. 
len den Opferstatus bestimmter Personen, sofern sich besagte Beschränkungen nur mittelbar - quasi als Kollateralschaden - für sie auswirkten. ${ }^{39}$

Im Hinblick auf Sperr-/Löschverfügungen im Internet hat der Gerichtshof bislang mehrfach differenzieren müssen. In der Rechtssache „Ahmet Yildirim v. Turkey“ bejahte er den Opferstatus des Beschwerdeführers, da dieser wegen einer umfassenden Sperrung des Zugriffs auf den Dienst „Google Sites“ wegen des Vorwurfs rechtswidriger Inhalte auf Websites Dritter nicht mehr im Stande war, seine eigene Website zu nutzen. ${ }^{40}$ Ebenso positiv entschied der Gerichtshof in der Rechtssache „Cengiz and Others v. Turkey“, da die Beschwerdeführer sowohl aktiv als auch passiv intensiv Gebrauch von den Informationsangeboten des vollständig blockierten Dienstes „YouTube“ machten und diese wegen ihrer Spezifika alternativlos seien. ${ }^{41}$ In der Rechtssache ,Akdeniz c. Turquie“ hingegen verneinte der EGMR den Opferstatus des Beschwerdeführers. Von der Sperre zweier Websites zur Verbreitung von Musik (,MySpace“ und „last.fm“) sah der Gerichtshof zum einen jeden Nutzer gleichsam betroffen; zum anderen berücksichtigte er, dass die begehrten, wenig bedeutsamen Informationen auch anderweitig hätten beschafft werden können. ${ }^{42}$

Den genannten Rechtssachen ist gemein, dass die Beeinträchtigungen der Beschwerdeführer als Nebenfolgen exzessiver staatlicher Maßnahmen zur Sperrung rechtswidriger Inhalte Dritter auftraten. In diesen Sonderkonstellationen nur mittelbarer Betroffenheit stellte der EGMR bei der Bewertung der Umstände des Einzelfalls auf Inhalte und Stellenwert des jeweils gesperrten Dienstes, die Nutzungsgewohnheiten der Beschwerdeführer und potenzielle Ausweichmöglichkeiten ${ }^{43}$ ab. Diese Fortentwicklung gegenüber der Rechtsprechung im analogen Bereich ist angesichts der Funktionslogiken des Internets folgerichtig, lässt aber zugleich noch einige Fragen offen: Wird man rein passive Rezipienten bestimmter

39 EGMR, 6.11.2001 - 40150/98 u.a. - Tanrikulu, Cetin, Kaya et autres c. Turquie, zu Interviewpartnern und Lesern einer ,verbotenen“ Zeitung; anders dagegen bei Aussagen eines Interviewpartners als Anlass für Maßnahmen EGMR, 8.10.2019 - 15449/09, Rn. 34 ff. - Margulev v. Russia.

40 EGMR, 18.12.2012 - 3111/10, Rn. 51 ff. - Ahmet Yildirim v. Turkey; vgl. auch EGMR, 23.6.2020 - 10795/14, Rn. 27, 35 - Vladimir Kharitonov v. Russia, wo der Gerichtshof auf das entsprechende Vorbringen der russischen Regierung zu Recht nicht mehr gesondert einging.

41 EGMR, 1.12.2015 - 48226/10 und 14027/11, Rn. 49 ff. - Cengiz and Others v. Turkey = NJOZ 2017, 1214, insb, Rn. 52: „political content ignored by the traditional media is often shared via YouTube, thus fostering the emergence of citizen journalism. From that perspective, the Court accepts that YouTube is a unique platform on account of its characteristics, its accessibility and above all its potential impact, and that no alternatives were available to the applicants". Dabei scheint der EGMR seine Anforderungen an die Relevanz einzelner Dienste jedoch nicht allzu restriktiv ausgestalten zu wollen, s. EGMR, 18.12.2012 - 3111/10, Rn. 54 - Ahmet Yildirim v. Turkey.

42 EGMR, 11.3.2014-20877/10, Rn. 24-Akdeniz c. Turquie.

43 Jüngst hat der Gerichtshof implizit erkennen lassen, dass das Argument, im Internet leicht auf andere Informations- und Kommunikationswege ausweichen zu können, zumindest nicht gegenüber den unmittelbaren Adressaten von Sperr-/Löschverfügungen verfängt, EGMR, 30.4.2019 48310/16 und 59663/17, Rn. 78, 84 - Kablis v. Russia, betr. das VKontakte-Profil und Blogeinträge des Beschwerdeführers. 
Dienste, Unterangebote ${ }^{44}$ oder gar einzelner Inhalte ${ }^{45}$ im Internet auch als „Opfer“ von Sperr- und Löschverfügungen erachten müssen? Welche Dienste oder Angebote sind wegen ihrer Spezifika für jeden Nutzer als entsprechend relevant, gar ,alternativlos“ einzustufen? Ist jemand weniger schutzwürdig, der mithilfe der Verlinkungstechniken des Internets ${ }^{46}$ gesperrte Inhalte Dritter in eigene Angebote integriert hat? Der EGMR steht vor der komplexen Herausforderung, die neuen Funktionsmechanismen verschiedenster Kommunikationsund Informationsdienste differenziert in Parameter seiner wertenden Einzelbetrachtung zu transponieren. Als letzte (und bisweilen einsame) Bastion des Grundrechtsschutzes in Europa sollte er die Anwendung dieses Ausschlusskriteriums im Interesse der Schutzsuchenden und ihrer neu gewonnen Freiheiten im Internet auf ein absolut notwendiges Minimum an Sachverhaltskonstellationen reduzieren.

(2) Rechtfertigungsanforderungen an staatliche Sperr- und Löschverfügungen

Ungleich stärker als Zweifel am Opferstatus dürften zukünftig indes Fragen zu den Rechtfertigungsanforderungen an staatliche Sperr- und Löschverfügungen in den Fokus der Rechtsprechung des EGMR rücken.

Bislang ist der Gerichtshof in allen einschlägigen Entscheidungen bereits von einer Verletzung des Art. 10 EMRK mangels Einhaltung der Vorgaben des Gesetzesvorbehalts ausgegangen. ${ }^{47}$ Dieser setzt über die bloße Existenz einer rechtlichen Grundlage hinaus auch deren öffentliche Zugänglichkeit (,accessability"), die Vorhersehbarkeit der mit ihrer Anwendung verbundenen Folgen (,foreseeability") und ihre Vereinbarkeit mit rechtsstaatlichen Grundsätzen („,compatibility with the rule of law“) (im Übrigen) voraus. ${ }^{48}$ In den bereits erwähnten Rechtssachen „Ahmet Yildirim v. Turkey“ und „Cengiz and Others v. Turkey“ monierte der Gerichtshof nicht nur den Mangel an Vorhersehbarkeit des türkischen Rechts, das (potenziell notwendige) Sperrungen unter Inkaufnahme weitreichender Kollateralblockaden rechtmäßiger Inhalte und gar ganzer Dienste (wie „Google Sites“ oder „YouTube“) schlicht nicht erfasste. ${ }^{49}$ Er kritisierte auch die Defizite der im Falle präventiver

44 Bspw. einzelne Google Sites, YouTube-Kanäle oder Facebook-Seiten.

45 Bspw. einzelne Blogeinträge, YouTube-Videos oder Facebook-Posts oder -Kommentare.

46 Bspw. Embedding, Hyperlinking und Sharing. S. zu Hyperlinking auch noch II. 3. a).

47 Wegen der daraus regelmäßig resultierenden Rechtsschutzdefizite erkannte der EGMR in Fällen betr. den russischen Gesetzesrahmen - 23.6.2020 - 20159/15, Rn. 41 ff. - Bulgakov v. Russia; 23.6.2020 - 61919/16, Rn.36ff. - Engels v. Russia; 23.6.2020 - 12468/15, 23489/15 und 19074/16, Rn. 46 ff. - OOO Flavus and Others v. Russia; 23.6.2020 - 10795/14, Rn. 48 ff. Vladimir Kharitonov v. Russia - jüngst zu Recht auch auf eine zusammenhängende Verletzung von Art. 13 EMRK.

48 S. dazu näher Brings-Wiesen, in: Spindler/Schuster, Erster Teil. A. Rn. 37-39.

49 EGMR, 18.12.2012 - 3111/10, Rn. 59-62, 67 - Ahmet Yildirim v. Turkey; 1.12.2015 - 48226/10 und 14027/11, Rn. 61, 65 - Cengiz and Others v. Turkey = NJOZ 2017, 1214; vgl. zur potenziellen Notwendigkeit der Etablierung eines internetspezifischen Regulierungsrahmens bereits EGMR, 5.5.2011 - 33014/05, Rn. 63 - Editorial Board of Pravoye and Shtekel v. Ukraine. 
Einschränkungen (,,prior restraints") besonders gebotenen rechtsstaatlichen Sicherungsmechanismen, konkret die unzureichende gesetzliche Einhegung und justizielle Kontrolle der staatlichen Entscheidungen. ${ }^{50}$

Vergleichbare Defizite identifizierte der EGMR auch im russischen Recht. Auch in den Rechtssachen „Kablis v. Russia“, „Bulgakov v. Russia“, „Engels v. Russia“, „OOO Flavus and Others v. Russia“ und „Vladimir Kharitonov v. Russia“ waren die Beschwerdeführer Betroffene exzessiver Sperrverfügungen geworden, in deren Folge ihre gesamten Onlinepräsenzen wegen des Vorwurfs der Illegalität einzelner eigener oder fremder Inhalte gesperrt wurden. ${ }^{51}$ In allen Verfahren gelangte der Gerichtshof zu dem Schluss, dass verschiedene Vorschriften des anwendbaren Gesetzes den zuständigen Behörden zu viel interpretatorischen Spielraum beließen ${ }^{52}$ und eine effektive gerichtliche Kontrolle - aus diesem Grund $^{53}$ und wegen fehlender Rechtsschutzgarantien zugunsten der betroffenen Anbieter ${ }^{54}$ - nicht gewährleistet war. Deutlicher als zuvor ${ }^{55}$ äußerte sich der Gerichtshof in diesen Verfahren auch zur Unverhältnismäßigkeit der Maßnahmen: Allein die Missachtung des Ver-

50 EGMR, 18.12.2012 - 3111/10, Rn. 63-68 - Ahmet Yildirim v. Turkey (s. zu den Anforderungen konkretisierend die Concurring Opinion of Judge Pinto de Albuquerque); 1.12 .2015 - 48226/10 und 14027/11, Rn. 62-65 - Cengiz and Others v. Turkey = NJOZ 2017, 1214.

51 EGMR, 30.4.2019 - 48310/16 und 59663/17, Rn. 84, 94 - Kablis v. Russia: Sperrung des VKontakte-Profils wegen eines einzelnen Posts (neben der Verfügung zur Löschung einzelner Blogeinträge); 23.6.2020 - 20159/19, Rn. 29, 33 f. - Bulgakov v. Russia: Sperrung der gesamten Website wegen einzelner abrufbarer Dateien (auch nach deren Entfernung); 23.6.2020 - 12468/15, 23489/15 und 19074/16, Rn. 29 - OOO Flavus and Others v. Russia: Sperrungen gesamter Websites wegen einzelner Inhalte (teils auch nach deren Entfernung); leicht anders in 23.6.2020 61919/16, Rn. 25 - Engels v. Russia: Löschung einer Unterseite vor der angedrohten Sperrung der gesamten Website; grundlegend anders in 23.6.2020 - 10795/14, Rn. 34-36, 39 - Vladimir Kharitonov v. Russia: Sperrung einer Website nur wegen geteilter IP-Adresse mit einem anderen Anbieter von als illegal eingestuften Inhalten.

52 EGMR, 30.4.2019 - 48310/16 und 59663/17, Rn. 86, 93-95 - Kablis v. Russia; 23.6.2020 20159/19, Rn. 32-34, 37 - Bulgakov v. Russia; 23.6.2020 - 61919/16, Rn. 27-30, 33 - Engels v. Russia; 23.6.2020 - 12468/15, 23489/15 und 19074/16, Rn. 31, 33-35, 40 f. - OOO Flavus and Others v. Russia; vgl. auch EGMR, 23.6.2020 - 10795/14, Rn. 38-42, 43 - Vladimir Kharitonov v. Russia.

53 EGMR, 30.4.2019 - 48310/16 und 59663/17, Rn. 96 - Kablis v. Russia; 23.6.2020 - 61919/16, Rn. 31 - Engels v. Russia; 23.6.2020 - 12468/15, 23489/15 und 19074/16, Rn. 43 - OOO Flavus and Others v. Russia.

54 Sie wurden weder über die Einleitung von Verfahren, den Vollzug von Maßnahmen oder deren rechtliche Begründung informiert, noch wurde ihnen (anders als den ISP) in diesen Verfahren die Möglichkeit zur Stellungnahme gegeben, s. EGMR, 23.6.2020 - 20159/15, Rn. 35-37 - Bulgakov v. Russia; 23.6.2020 - 61919/16, Rn. 31 f. - Engels v. Russia; 23.6.2020 - 10795/14, Rn. 43 f. Vladimir Kharitonov v. Russia; insb. betr. journalistische Websites 23.6.2020 - 12468/15, 23489/15 und 19074/16, Rn. 40, 42 - OOO Flavus and Others v. Russia; vgl. zum Fehlen einer Eilrechtsschutzoption auch EGMR, 30.4.2019 - 48310/16 und 59663/17, Rn. 96 - Kablis v. Russia.

55 Der EGMR sah sich hier vereinzelt gezwungen, die einzelnen Voraussetzungen der Rechtfertigung kumuliert zu prüfen, 30.4.2019 - 48310/16 und 59663/17, Rn. 85 - Kablis v. Russia; 23.6.2020 12468/15, 23489/15 und 19074/16, Rn. 29 - OOO Flavus and Others v. Russia. Ähnlich deutete er 
fahrens zur Genehmigung einer risikoarmen Demonstration hielt er in „Kablis v. Russia“ für nicht tragfähig, um die Sperrung dreier Blogeinträge und des „VKontakte“-Profils des Beschwerdeführers zu rechtfertigen, mit denen (partiell) zu einer Teilnahme aufgerufen wurde. ${ }^{56}$ In eine ähnliche Richtung argumentierte der EGMR in einem obiter dictum in „Engels v. Russia“ hinsichtlich der Verbreitung von Informationen über Programme zur Umgehung von Internetfiltern und setzte damit (mittelbar) ein starkes Zeichen für ein freies Internet: Deren Einsatz diene nicht ausschließlich der Verfolgung illegaler Zwecke, vielmehr seien sie wie alle Informationstechnologien zunächst inhaltsneutral, sodass ein Verbot, über sie zu informieren, nicht ohne besondere Rechtsgrundlage ergehen dürfe. ${ }^{57}$ Besonders deutlich stellte der Gerichtshof indes in „OOO Flavus and Others v. Russia“ klar, dass jede exzessive Sperrverfügung, in deren Folge ohne nähere Darlegung von Ziel und Notwendigkeit auch legale Inhalte in Mitleidenschaft gezogen würden, nicht zu rechtfertigen sei. $^{58}$

In Anbetracht des Stellenwerts des Internets für den Kommunikations- und Informationsprozess ist zu begrüßen, dass der EGMR zunächst besonders strenge Anforderungen an die speziellen Rechtsrahmen und Rechtsschutzmechanismen betreffend staatliche Sperrund Löschverfügungen stellt. Es steht indes zu vermuten, dass zukünftige Entscheidungen ${ }^{59}$ vermehrt auch Fragen legitimer Eingriffszwecke und der Verhältnismäßigkeit (mit Blick auf Anlass, Reichweite ${ }^{60}$ und Wirkung ${ }^{61}$ einzelner Verfügungen unter Berücksichtigung der Funktionscharakteristika des Internets) problematisieren werden. Damit werden unweigerlich auch kontroverse materielle Fragestellungen in den Vordergrund rücken und den Gerichtshof (auf ein Neues) vor die Herkulesaufgabe stellen, Resilienz und Akzeptanz der

bereits in 18.12.2012 - 3111/10, Rn. 66 - Ahmet Yildirim v. Turkey, eine Unverhältnismäßigkeit der Maßnahme an.

56 EGMR, 30.4.2019 - 48310/16 und 59663/17, Rn. 98-105 - Kablis v. Russia; vgl. jüngst auch EGMR, 23.6.2020 - 12468/15, 23489/15 und 19074/16, Rn. 33 f. - OOO Flavus and Others v. Russia.

57 EGMR, 23.6.2020 - 61919/16, Rn. 28-30 - Engels v. Russia.

58 EGMR, 23.6.2020 - 12468/15, 23489/15 und 19074/16, Rn. 38 - OOO Flavus and Others v. Russia; vgl. auch EGMR, 23.6.2020 - 10795/14, Rn. 46 - Vladimir Kharitonov v. Russia.

59 Aktuell sind zahlreiche Verfahren zu diesem Problembereich anhängig, die die Rechtsprechung weiter präzisieren und diversifizieren dürften: 33421/16 - Klimova v. Russia; 36589/17 - AZADLIG.INFO and Others v. Azerbaijan; 35550/18 - Engels v. Russia; 56491/18 und 11884/19 - Navalny v. Russia and OOO ZP v. Russia; 25479/19 - Wikimedia Foundation, Inc. v. Turkey; 55496/19 - OOO Mediafokus v. Russia; 15355/14 - Krysztofiak v. Poland.

60 Dabei können technische Möglichkeiten eine Rolle spielen, s. EGMR, 1.12.2015 - 48226/10 und 14027/11, Rn. 17, 23, 46, 64 - Cengiz and Others v. Turkey = NJOZ 2017, 1214.

61 Dabei dürfte sich die Frage stellen, ob wie schon in anderen Zusammenhängen die Verfügbarkeit anderer Informations- und Kommunikationswege wirklich eine Rolle spielen kann, vgl. EGMR, 28.6.2001 - 24699/94, Rn. 77 - VgT Verein gegen Tierfabriken v. Switzerland; (GK) 13.7.2012 16354/06, Rn. 73-75 - Mouvement raëlien suisse v. Switzerland; (GK) 22.4.2013 - 48876/08, Rn. 124 - Animal Defenders International v. UK: ,access to alternative media is key to the proportionality of a restriction on access to other potentially useful media". 
Schutzstandards unter Art. 10 EMRK in den (rechts-)kulturell diversifizierten Konventionsstaaten sicherzustellen, ohne bereits auf einen europäischen Konsens aufbauen zu können. Exemplarisch sei hier nur auf das europäische Ringen um den Umgang mit Desinformationen verwiesen. ${ }^{62}$ Während über das Kernanliegen weitgehend Einigkeit besteht, wird man Gleiches nicht für konkrete Fragestellungen - wie bereits die Bestimmung des Kreises problematischer Inhalte (insbesondere in Grenzbereichen) - behaupten können. Insofern wird der EGMR unter Berücksichtigung konventionsstaatlicher Spielräume in vielen Bereichen zunächst nur Mindeststandards etablieren können.

\section{bb) Gleiches Recht für alle? Restriktionen der Internetnutzung im Strafvollzug}

Die Bedeutsamkeit des Internets reanimiert und evolviert auch die Diskussion um die besondere Streitfrage des Medienzugangs im Strafvollzug. Gewisse Beschränkungen von Kommunikation und Information werden wegen der Prävention gegen etwaige Sicherheitsrisiken und einem potenziell erhöhten Kosten- und Personalaufwand grundsätzlich akzeptiert. ${ }^{63}$ Hinsichtlich des „Ob“ und „Wie“ der Nutzung des World Wide Web scheinen indes einige Konventionsstaaten noch nicht das richtige Maß gefunden zu haben. Der Gerichtshof hat in bislang zwei zentralen Entscheidungen das Recht von Strafgefangenen auf Zugang zu Informationen im Internet aus Art. 10 EMRK $^{64}$ durch Fokussierung einer strengen Einzelfallprüfung im Ergebnis gestärkt, sich dabei jedoch für die Zukunft einen denkbar weiten Abwägungsspielraum erhalten.

In der Rechtssache „Kalda v. Estonia“65 war dem Beschwerdeführer der für die Zwecke der persönlichen Prozessvorbereitung gewünschte Zugang zu den Websites des Informationsbüros des Europarats sowie des Justizkanzlers und des Parlaments von Estland versagt worden; in der Rechtssache „Jankovskis v. Lithuania“66 hatte man dem Antragssteller, der sich über die Möglichkeiten der Aufnahme eines universitären (Fern-)Studiums informieren wollte, in der Strafanstalt den Zugriff auf die einschlägige Website des litauischen Ministe-

62 S. zum „weichen“ Ansatz der Europäischen Kommission unter: https://ec.europa.eu/digital-singlemarket/en/tackling-online-disinformation; vgl. auch die laufend aktualisierte Darstellung des Poynter Institute zu weltweit verschiedenen Ansätzen unter: https://www.poynter.org/ifcn/anti-mis information-actions/.

63 S. nur Esser, NStZ 2018, 121 (125).

64 Deren Zulässigkeit wurde allerdings auch unter Erwägung etwaiger Risiken von Kommunikationsmöglichkeiten mit der Außenwelt über das Internet diskutiert. Den Wunsch einer aktiven Teilnahme am Kommunikationsprozess über das Internet wird man am Maßstab von Art. 10 EMRK noch einmal eigenständig bewerten müssen. S. abgrenzend auch EGMR, 18.6.2019 - 47121/06 u.a., Rn. 42, 59 - Mehmet Reşit Arslan et Orhan Bingöl c. Turquie, für eine Prüfung am Maßstab des Rechts auf Bildung gemäß Art. 2 ZP II zur EMRK.

65 EGMR, 19.1.2016 - 17429/10 - Kalda v. Estonia = NJOZ 2018, 1598.

66 EGMR, 17.1.2017 - 21575/08 - Jankovskis v. Lithuania = NJOZ 2018, 1158, dazu Bode, ZIS 2017, $348 \mathrm{ff}$. 
riums für Bildung und Wissenschaft verweigert. ${ }^{67}$ In beiden Entscheidungen hielt der EGMR zunächst fest, aus Art. 10 EMRK folge keine generelle Pflicht der Staaten, Gefangenen Zugang zum Internet oder zu bestimmten Websites zu gewähren. ${ }^{68}$ Letztlich bejahte er jedoch zumindest aufgrund der konkreten Umstände zweimal eine Verletzung der Informationsfreiheit wegen Unverhältnismäßigkeit ${ }^{69}$ : Beide Betroffenen verfolgten mit ihren Zugangsbegehren legitime und wichtige Zwecke, ${ }^{70}$ konnten die erwünschten Informationen (ohne unangemessene Hürden) nur im Internet abrufen ${ }^{71}$ und wurden staatlicherseits ohne konkrete Begründung bezüglich eines erhöhten Sicherheitsrisikos oder Aufwands abgewie$\operatorname{sen}^{72}$. Kalda kam darüber hinaus zugute, dass das estnische Recht bereits den Zugang zu ausgewählten Websites über speziell ausgerüstete Computer und unter Aufsicht erlaubte. ${ }^{73}$

Obgleich der EGMR eine generelle Verpflichtung zur Bereitstellung eines Internetzugangs ausdrücklich ablehnte und auch die grundsätzliche Relevanz von Sicherheits- und wirtschaftlichen Bedenken wiederholt betonte ${ }^{74}$, intensivierte er die Rechtfertigungsanforderungen. Selbst wo im nationalen Recht überhaupt kein Anspruch auf Zugang zum Internet vorgesehen ist, kann dessen Ablehnung im Einzelfall konventionswidrig sein. ${ }^{75}$ Dies ist insbesondere dann denkbar, wenn für das Individuum relevante Informationen (in einer be-

67 Zu beiden Entscheidungen Esser, NStZ 2018, 121 ff.; Knauer, Die Rechtsprechung des EGMR zur Nutzung des Internets im Strafvollzug, 2018, S. 43 ff.

68 EGMR, 19.1.2016 - 17429/10, Rn. 45 - Kalda v. Estonia = NJOZ 2018, 1598; 17.1.2017 21575/08, Rn. 55 - Jankovskis v. Lithuania = NJOZ 2018, 1158.

69 S. auch die vergleichbaren Defizite in EGMR, 18.6.2019 - 47121/06 u.a., Rn. 55-72 - Mehmet Reșit Arslan et Orhan Bingöl c. Turquie, unter Berücksichtigung der Rechtsprechung zu Art. 10 EMRK.

70 EGMR, 19.1.2016 - 17429/10, Rn. 50 - Kalda v. Estonia = NJOZ 2018, 1598: Rechtsschutz; 17.1.2017 - 21575/08, Rn. 59 - Jankovskis v. Lithuania = NJOZ 2018, 1158: Weiterbildung als sinnstiftende Tätigkeit und zwecks Resozialisierung.

71 EGMR, 19.1.2016 - 17429/10, Rn. 51 - Kalda v. Estonia = NJOZ 2018, 1598: estnische Übersetzung der EGMR-Rechtsprechung; 17.1.2017 -21575/08, Rn. 60, $62-$ Jankovskis v. Lithuania $=$ NJOZ 2018, 1158: sich ständig verändernde Informationen zu Studienangeboten; in beiden Fällen wies der Gerichtshof gezielte Auskunftsverlangen als Alternativen zur Website-Recherche als inadäquat zurück.

72 EGMR, 19.1.2016 - 17429/10, Rn. 53 - Kalda v. Estonia = NJOZ 2018, 1598; 17.1.2017 21575/08, Rn. 61 f. - Jankovskis v. Lithuania $=$ NJOZ 2018, 1158, wo auch der Aspekt der Weiterbildung gar nicht weiter berücksichtigt wurde.

73 EGMR, 19.1.2016 - 17429/10, Rn. 21, 45, 49, 53 - Kalda v. Estonia = NJOZ 2018, 1598; krit. dazu die Dissenting Opinion of Judge Kjølbro, Rn. 2, 7 f. Weniger klar war die litauische Rechtslage, s. EGMR, 17.1.2017 - 21575/08, Rn. 10, 12, 14-16, 19, 29-34, 55 - Jankovskis v. Lithuania = NJOZ 2018, 1158; s. auch a.a.O., Rn. 57, zu entsprechend diskussionsbedürftigen Ausführungen betr. den Gesetzesvorbehalt.

74 EGMR, 19.1.2016 - 17429/10, Rn. 53 - Kalda v. Estonia = NJOZ 2018, 1598; 17.1.2017 21575/08, Rn. 61 - Jankovskis v. Lithuania = NJOZ 2018, 1158.

75 EGMR, 17.1.2017 - 21575/08, Rn. 29-34, 55 - Jankovskis v. Lithuania = NJOZ 2018, 1158. 
stimmten Aufbereitung ${ }^{76}$ ) nur online verfügbar ${ }^{77}$ oder durch eine entsprechende WebsiteRecherche ermittelbar ${ }^{78}$ sind und die genannten Bedenken nicht einzelfallbezogen konkretisiert werden (können $)^{79}$. Diese Aspekte würdigte der Gerichtshof nicht zuletzt unter Berücksichtigung einer universellen Tendenz im Völkerrecht zur Anerkennung eines allgemeinen Rechts auf Internetzugang. ${ }^{80}$ Vor diesem Hintergrund erscheint die Negation einer generellen Verpflichtung zur Bereitstellung eines Internetzugangs bereits jetzt deutlich relativiert. Zukünftige Judikate ${ }^{81}$ dürften diese progressive Entwicklungslinie - insbesondere im Interesse der individuellen Selbstverwirklichung der Betroffenen - eher fortführen und angesichts der fundamentalen Bedeutung der Konfliktlage und der bislang nicht abschließend geklärten Fragen eines allgemeinen Rechts auf Internetzugang und seiner Tragweite im Strafvollzug letztlich eine Leitentscheidung der Großen Kammer provozieren ${ }^{82}$. Bereits jetzt sind die Judikate jedoch als Appell an die Mitgliedstaaten zu deuten, einschlägige Normkomplexe liberalisierend anzupassen.

Mit großem Interesse wird man daher auch die Auswirkungen der Rechtsprechung auf die fortwährende Fachdiskussion in Deutschland beobachten müssen. ${ }^{83}$ Erste verfassungsgerichtliche Beschlüsse lassen eine Rezeption (deutlich) erkennen. ${ }^{84}$

76 EGMR, 17.1.2017 - 21575/08, Rn. 60, 62 - Jankovskis v. Lithuania = NJOZ 2018, 1158: umfassende und sich ständig verändernde Informationen über landesweite Bildungsmöglichkeiten.

77 EGMR, 19.1.2016 - 17429/10, Rn. 51 f. - Kalda v. Estonia = NJOZ 2018, 1598.

78 EGMR, 19.1.2016 - 17429/10, Rn. 50 - Kalda v. Estonia = NJOZ 2018, 1598; 17.1.2017 21575/08, Rn. 60 - Jankovskis v. Lithuania = NJOZ 2018, 1158.

79 EGMR, 19.1.2016 - 17429/10, Rn. 53 - Kalda v. Estonia = NJOZ 2018, 1598; 17.1.2017 21575/08, Rn. 61 - Jankovskis v. Lithuania = NJOZ 2018, 1158. Völlig unbeleuchtet blieben daher bislang die technischen Hintergründe von Sicherheitsrisiken und -maßnahmen bei der Bereitstellung von Internetzugängen.

80 EGMR, 19.1.2016 - 17429/10, Rn. 22-25, 52 - Kalda v. Estonia = NJOZ 2018, 1598; 17.1.2017 21575/08, Rn. 35 f., 62 - Jankovskis v. Lithuania $=$ NJOZ 2018, 1158. S. zu dieser umstrittenen Fragestellung Tully, HRLR 2014, 175 ff.; Aguerre, Right of access to the internet - global approaches, 2019, S. 31 ff.; vgl. zur Rechtslage nach dem GG Oermann, Gewährleistung der Möglichkeit internetbasierter Kommunikation, 2018, passim.

81 Anhängig ist aktuell die Rechtssache 68550/17 - Demir c. Turquie.

82 S. bereits die Dissenting Opinion of Judge Kjølbro zu EGMR, 19.1.2016 - 17429/10 - Kalda v. Estonia, Rn. 15.

83 S. dazu aus jüngerer Zeit nur Bode, ZIS 2017, 348 (351 ff.); ders., FS 2018, 219 ff.; ders., NK 2019, 30 ff.; sowie Esser, NStZ 2018, 121 ff., jeweils m. zahlr. w. Nw.

84 BVerfG, 27.3.2019 - 2 BvR 2268/18, NJW 2019, 1738 (Rn. 12), zu dessen Signalwirkung Bode, NStZ-RR 2019, 192; SächsVerfGH, 27.6.2019 - 64-IV-18, NStZ-RR 2019, 292 (294 f.), dazu Bode, NStZ-RR 2019, 295 f., und Esser, NStZ 2020, 107 ff. 


\section{b) Die Wirkkraft im Internet}

Die zentralen Charakteristika des Internets tragen nicht bloß zu dessen Bedeutungszuwachs für den menschlichen Kommunikations- und Informationsprozess an sich bei, sondern prägen auch dessen für die rechtliche Bewertung mitentscheidende Wirkkraft.

Der EGMR verfolgt seit jeher den Ansatz, die Wirkweisen verschiedener Kommunikations- und Informationsmittel in seiner Prüfung zu berücksichtigen. ${ }^{85}$ Dabei hat er auch eine Wirkungshierarchie der Medien entwickelt. Während er in ständiger Rechtsprechung eine geringere Wirkkraft von Print- gegenüber audiovisuellen Medien erkennt, ${ }^{86}$ nimmt das Internet an sich einen Zwischenrang ein. Die strikteren Vorschriften des britischen Rechts betreffend politische Werbung in Radio und Fernsehen akzeptierte der Gerichtshof noch im Jahr 2013 mit der Begründung, dass trotz ihres Bedeutungszuwachses - zumindest im Vereinigten Königreich - noch keine dem Rundfunk vergleichbare Wirkkraft von „Internet und sozialen Medien“ nachweisbar gewesen sei. Dabei stellte er die unmittelbare Wirkkraft von linearem Bild und Ton im eigenen Zuhause den der Nutzung des Internets und sozialer Medien inhärenten Wahlmöglichkeiten gegenüber. ${ }^{87}$ Kontrastierend stufte er das Schadensrisiko für andere Grundrechte durch die Kommunikation im Internet ohne Zweifel höher ein als das der Printmedien. ${ }^{88}$

Abgesehen davon, dass einige dieser Annahmen des EGMR durchaus diskussionsbedürftig sind, kann der Ansatz einer pauschalen Verortung des „Internets“ in einer Wirkungshierarchie der Medien ${ }^{89}$ nur die Spitze des Eisbergs einer komplexen Herausforderung sein. Die Diversität von Internetdiensten und deren Funktions- und Wirkungsmechanismen verlangt nach einer deutlich detailorientierteren Betrachtung (im jeweiligen Einzelfall). ${ }^{90}$ Erste Anzeichen für eine entsprechend sensibilisierte Herangehensweise finden sich in der Judikatur bereits.

In der Rechtssache „Wrona v. Poland“ stellte der EGMR fest, dass diffamierende Aussagen in einem zugangsbeschränkten, privaten Online-Forum nur eine begrenzte Reichwei-

85 S. dazu m. w. Nw. Brings-Wiesen, in: Spindler/Schuster, Erster Teil. A. Rn. 56.

86 EGMR (GK), 23.9.1994 - 15890/89, Rn. 31 - Jersild v. Denmark = NStZ 1995, 237; (GK) 16.6.2015 - 64569/09, Rn. 134 - Delfi AS v. Estonia = NJW 2015, 2863; 21.2.2017 - 42911/08, Rn. 109 - Orlovskaya Iskra v. Russia; noch wirkungsärmer gar einfache literarische Veröffentlichungen, s. EGMR (GK), 8.7.1999 - 23462/94, Rn. 48 - Arslan v. Turkey.

87 EGMR (GK), 22.4.2013 - 48876/08, Rn. 119 - Animal Defenders International v. UK. Die Validität dieses Befunds wird man heute zumindest kritisch hinterfragen müssen, so bereits O'Leary, Irish Jurist 2018, 59 (71).

88 EGMR, 5.5.2011 - 33014/05, Rn. 63 - Editorial Board of Pravoye Delo and Shtekel v. Ukraine; 4.12.2018 - 11257/16, Rn. 66 - Magyar Jeti Zrt v. Hungary = NJW 2019, 3201. Vgl. auch EGMR, 22.4.2010 - 40984/07, Rn. 95 - Fatullayev v. Azerbaijan.

89 In den meisten Fällen dürfte die Frage der Wirkungshierarchie unterschiedlicher Medien ohnehin nur auf abstrakter gesetzlicher Ebene eine Rolle spielen.

90 Deutlich in diese Richtung zul. auch das BVerfG, 6.11.2019 - 1 BvR 16/13, K\&R 2020, 51 (Rn. 125) - Recht auf Vergessen I. 
te gehabt hätten. ${ }^{91}$ In vergleichbarer Weise berücksichtigte er in der Rechtssache „Tamiz v. $U K^{\prime \prime}$ für die Erheblichkeit einer ehr- und rufverletzenden Wirkung von Kommentaren zu einem Artikel auf einem offen zugänglichen, privaten Blog, dass die Reichweite einzelner in der Flut von Millionen von Nutzern täglich veröffentlichter Kommentare begrenzt sei. ${ }^{92}$ Demgegenüber würdigte der Gerichtshof in seiner Entscheidung zur Rechtssache „Beizaras and Levickas v. Lithuania“ entgegen des Vorbringens der litauischen Regierung, Einträge auf „Facebook“ erzeugten - anders als auf Nachrichtenportalen - nur wenig Aufmerksamkeit, nicht nur, dass bereits ein einzelner Hasskommentar ernst zu nehmen sei, sondern darüber hinaus auch, dass das betroffene Bild vorher „,viral gegangen“ war. ${ }^{93}$ Einem vermeintlichen Hetzkommentar auf einem nur mäßig frequentierten Blog sprach der EGMR in der Rechtssache „Savva Terentyev v. Russia“ hingegen eine nur geringe Wirkkraft zu. ${ }^{94}$ Insgesamt zeigt sich also, dass der Gerichtshof bei der Bestimmung der Reichweite von Angeboten deren Relevanz für situative und thematische Teilöffentlichkeiten berücksichtigt. ${ }^{95}$

Obgleich der Faktor „Reichweite“ für den EGMR eine derart entscheidende Rolle spielt, befindet sich die gerichtliche Begutachtung der für die Sichtbarkeit von Inhalten so wichtigen Suchmaschinen ${ }^{96}$ erst in einem Anfangsstadium: In der Rechtssache „Cicad c. Suisse" berücksichtigte der EGMR, dass unzureichend belegte Behauptungen über den Betroffenen nicht nur über eine Website, sondern durch die bloße Eingabe seines Namens in eine Suchmaschine selbst ohne deren Aufruf für eine große Personenzahl verfügbar waren. ${ }^{97}$ In der Rechtssache „M.L. and W.W. v. Germany“ erkannte der Gerichtshof an, dass es

91 EGMR, 12.12.2017 - 68561/13, Rn. 21, 23 - Wrona v. Poland; vgl. zur Besonderheit geschlossener Facebook-Gruppen die anhängige Rechtssache 16695/19 - Kozan c. Turquie.

92 EGMR, 19.9.2017 - 3877/14, Rn. 80 - Tamiz v. UK; in diese Richtung auch EGMR, 17.7.2018 31221/15, Rn. 38 f. - Egill Einarsson v. Iceland (No. 2).

93 EGMR, 14.1.2020 - 41288/15, Rn. 127 - Beizaras and Levickas v. Lithuania. Bemerkenswert ist auch, dass der EGMR, a.a.O., das allgemeine Ausmaß des Problems von Hate Speech online und in sozialen Netzwerken in Litauen mitberücksichtigte. Vgl. zur Reichweite über soziale Netzwerke auch EGMR, 16.7.2019 - 12200/08, 35949/11 und 58282/12, Rn. 84 - Zhdanov and Others v. Russia; dazu auch die Joint Partly Dissenting Opinion of Judges Keller, Serghides and Elósegui, Rn. $11 \mathrm{f}$.

94 EGMR, 28.8.2018 - 10692/09, Rn. 79 ff. - Savva Terentyev v. Russia.

95 S. allg. EGMR, 28.8.2018 - 10692/09, Rn. 79 - Savva Terentyev v. Russia: „It is therefore essential for the assessment of a potential influence of an online publication to determine the scope of its reach to the public"; vgl. für die Adressierung thematischer Teilöffentlichkeiten auch EGMR, 4.6.2020 - 31955/11, Rn. 55, 60 - Jezior c. Pologne.

96 Dementsprechend ist auch die Verantwortlichkeit von deren Betreibern gesondert in den Blick zu nehmen, s. dazu noch unter II. 2. a) bb).

97 EGMR, 7.6.2016 - 17676/09, Rn. 60 - Cicad c. Suisse. Die nicht minder interessante Frage der Wirksamkeit einer Gegendarstellung im Internet musste vom Gerichtshof nicht vertieft problematisiert werden, a.a.O., Rn. 61. 
primär an Suchmaschinen läge, dass Informationen für Internetnutzer auffindbar seien, ${ }^{98}$ und sie die Verbreitung von Inhalten verstärkten. ${ }^{99}$

Um zu einer konsistenten Methodik unter Berücksichtigung aller relevanten Wirkfaktoren zu gelangen, wird der EGMR zukünftig gefordert sein, nicht nur die Dynamik der informationstechnischen Progression und des sich wandelnden Nutzerverhaltens, sondern auch die sich kontinuierlich aktualisierenden und präzisierenden Erkenntnisse der Medienwirkungsforschung angemessen zu würdigen.

\section{Wie sind Rolle, Funktion und Arbeitsweise von (neuen) Akteuren im digitalen Informations- und Kommunikationsprozess einzuordnen?}

Besonderen Einfluss nimmt das Internet als Medium auch auf die am Kommunikationsund Informationsprozess beteiligten Akteure. Während völlig neue Rollenbilder entstanden sind und zentrale Aufmerksamkeit erlangt haben, sind Funktion und Arbeitsweise traditioneller Akteure durch ihr Wirken im digitalen Raum rechtlich neu gewürdigt worden. Dabei ist es notwendigerweise zur Anerkennung neuer und zur Modifikation bestehender Rechte und Pflichten gekommen.

\section{a) Das Internet und die journalistische Arbeit}

Da journalistisch tätigen Personen in ihrer Funktion als ,public watchdogs" traditionell eine hervorgehobene Stellung im Kommunikations- und Informationsprozess zukommt, ${ }^{100}$ ist von besonderem Interesse, wie sich ihr Wirken im Internet in der Rechtsprechung des EGMR niederschlägt.

Dabei ist zunächst festzustellen, dass der Gerichtshof an seinem Grundansatz der Annahme bestimmter Sonderrechte professioneller Journalisten festhält. ${ }^{101}$ In der Rechtssache „Editorial Board of Pravoye and Shtekel v. Ukraine“ hielt er für problematisch, dass die Wiedergabe von Informationen auf einer Website nicht von den Haftungsprivilegien für professionelle Journalisten nach dem ukrainischen Recht erfasst wurde. Der nationale

98 Für eine Auseinandersetzung mit der für die Auffindbarkeit relevanten Problematik der Personalisierung von Suchergebnissen hat sich bislang noch kein Anlass geboten.

99 EGMR, 28.6.2018 - 60798/10 und 65599/10, Rn. 97 - M.L. and W.W. v. Germany = NJW 2020, 295. Der Gerichtshof ging gar soweit, dies als wesentlichen Grund für die gegenüber der Presse gesteigerte Wirkkraft des Internets zu benennen, a.a.O., Rn. 91.

100 Ausdrückliche Erwähnung des Terminus erstmalig in EGMR, 25.3.1985 - 8734/79, Rn. 58 - Barthold v. Germany; s. zu dieser Rolle grundlegend nur Oster, Media Freedom as a Fundamental Right, 2015, passim; ders., Kommunikationsdeliktsrecht, 2019, S. 149 ff.; für die EMRK im Überblick Holoubek, AfP 2003, 193 (194ff.).

101 Dies gilt unverändert, soweit das Internet wie andere Kommunikationsmittel in die journalistische Arbeit einbezogen wird, vgl. nur zur Überwachung der Internetkommunikation EGMR, 13.9.2018 - 58170/13, 62322/14 und 24960/15, Rn. 487 ff. - Big Brother Watch and Others v. UK = NLMR 2018, 428 (Verfahren bei der GK anhängig). 
Rechtsrahmen ließ das Internet als mögliche Informationsquelle schlicht unberücksichtigt und behinderte aus Sicht des EGMR so die journalistische Arbeit. ${ }^{102}$

Noch deutlicher wird die Fortsetzung der Sonderbehandlung jedoch auf der Kehrseite des gehobenen Schutzstandards. Seit langem macht der EGMR diesen von der Einhaltung journalistischer Sorgfaltspflichten abhängig. ${ }^{103}$ In jüngerer Zeit hat der Gerichtshof wiederholt betont, dass er dieser Erwägung wegen des Einflusses der Medien in der modernen Gesellschaft und der enormen Mengen an Informationen, die von einer zunehmenden Zahl von Akteuren über traditionelle und elektronische Medien verbreitet werden, sogar noch mehr Bedeutung zumisst. ${ }^{104}$ Auch wenn die Auswirkungen dieser Rechtsprechung bislang kaum konkret fassbar sind, ist ihre Grundtendenz zu begrüßen: Professionelle journalistische Angebote können in der Informationsflut des Internets als „safe havens“ fungieren, ${ }^{105}$ zugleich aber befinden sie sich wegen der Dynamik der Online-Welt in einem unerbittlichen Sprint um die Aufmerksamkeit potenzieller Rezipienten, bei dem ein gesteigertes Risiko der Sorgfaltsreduktion besteht. Beide Aspekte rechtfertigen die Aufrechterhaltung besonderer Rechte und Pflichten professioneller Journalisten und deren Anpassung an die Funktionslogiken des Internets.

\section{aa) Die Trennung von Privatperson und Journalist}

Die Frage, ob Personen im Einzelfall tatsächlich in ihrer Funktion als Journalist wirken oder betroffen sind, beschäftigt den EGMR regelmäßig. ${ }^{106}$ In seiner Entscheidung der Rechtssache „Fatullayev v. Azerbaijan“ hielt der Gerichtshof es im Rahmen der Frage, ob Werturteile auf einer ausreichenden Quellenlage beruhten, für relevant, zu differenzieren, ob der unter seinem Klarnamen auftretende, bekannte Journalist in eben dieser Funktion oder als gewöhnlicher Bürger in einem Diskussionsforum agierte. ${ }^{107}$ Die Erwägung war zwar letztlich nicht entscheidungserheblich, verdeutlicht aber, dass Journalisten sich ihrer

102 EGMR, 5.5.2011 - 33014/05, Rn. 63 f. - Editorial Board of Pravoye and Shtekel v. Ukraine; vgl. auch EGMR, 16.7.2013 - 33846/07, Rn. 58 - Węgrzynowski and Smolczewski v. Poland = AfP 2014, 517; s. betr. die Haftung für einen Verweis auf einen vermeintlich diffamierenden Facebook-Post auch die anhängige Rechtssache 77940/17 - Index.hu ZRT v. Hungary.

103 Deutlich EGMR (GK), 21.1.1999 - 29183/95, Rn. 54 - Fressoz and Roire v. France = ZUMRD 1999, 161; s. bereits EGMR, 26.4.1995 - 15974/90, Rn. 37 - Prager and Oberschlick v. Austria.

104 EGMR (GK), 10.12.2007 - 69698/01, Rn. 104 - Stoll v. Switzerland = NJW-RR 2008, 1141; 28.3.2013 - 14087/08, Rn. 42 - Novaya Gazeta and Borodyanskiy v. Russia; s. auch EGMR, 4.12.2018 - 11257/16, Rn. 64 - Magyar Jeti Zrt v. Hungary = NJW 2019, 3201.

105 Vgl. zur Rolle des öffentlich-rechtlichen Rundfunks in Deutschland BVerfG, 18.7.2018 - 1 BvR 1675/16 u.a., BVerfGE 149, 222 (262, Rn. 80); dazu bereits Dörr/Holznagel/Picot, ZUM 2016, 920 (931, 940 f.).

106 Vgl. insbesondere EGMR, 16.7.2013 - 73469/10, Rn.99f. - Nagla v. Latvia; 13.10.2015 37428/06, Rn. 79 - Bremner v. Turkey = NJOZ 2016, 1509.

107 EGMR, 22.4.2010 - 40984/07, Rn. 94 f. - Fatullayev v. Azerbaijan; vgl. indes EGMR, 23.6.2015 -32297/10, Rn. 50 ff. - Niskasaari and Otavamedia Oy v. Finland. 
Doppelfunktion im Internet bewusst sein und dementsprechend Sorgfalt walten lassen sollten.

\section{bb) Die neuen , watchdogs“}

Bemerkenswert ist auch, dass der EGMR in jüngerer Zeit die Tendenz zeigt, die Exklusivität dieses Status aufzuweichen. Diese Entwicklung nahm ihren Ursprung mit der Anerkennung anderer ,watchdogs“. Der Gerichtshof geht davon aus, dass Vereinigungen der Zivilgesellschaft (bzw. deren Angehörige - als sog. ,,social watchdogs“"), ${ }^{108}$ Wissenschaftler ${ }^{109}$ und Schriftsteller ${ }^{110}$ durch ihre Tätigkeiten wie journalistische Medien öffentliche Debatten anstoßen und bereichern. Für diese Personen(-gruppen) verlangt der EGMR daher einen „vergleichbaren“ (,similar") Schutz unter der EMRK. Dessen Reichweite ist jedoch noch nicht abschließend geklärt. Der Gerichtshof ging bereichsweise bereits von einer Parallelisierung der Bewertungsmaßstäbe aus, ${ }^{111}$ während er an anderer Stelle eine Vergleichbarkeit verneinte $^{112}$. Wechselseitig hat der EGMR indes deutlich gemacht, dass der besondere Schutz auch für diese anderen ,watchdogs“ unter dem Vorbehalt stehen soll, dass sie im gu-

108 EGMR, 27.5.2004 - 57829/00, Rn. 42 - Vides Aizsardzības Klubs c. Lettonie (im Bereich des Umweltschutzes); 14.4.2009 - 37374/05, Rn. 27 - Társaság a Szabadságjogokért v. Hungary (im Bereich des Menschenrechtsschutzes); 28.11.2013 - 39534/07, Rn. 34 ff. - Österreichische Vereinigung zur Erhaltung, Stärkung und Schaffung eines wirtschaftlich gesunden land- und forstwirtschaftlichen Grundbesitzes v. Austria; (GK) 22.4.2013 - 48876/08, Rn. 103 - Animal Defenders International v. UK; 25.6.2013 - 48135/06, Rn. 20 - Youth Initiative for Human Rights v. Serbia; 17.2.2015 - 6987/07, Rn. 38, 41, 54 - Guseva v. Bulgaria (im Bereich des Tierschutzes); (GK) 8.11.2016 - 18030/11, Rn. 159, 164 ff. - Magyar Helsinki Bizottság v. Hungary = AfP 2017, 301 (im Bereich des Menschenrechtsschutzes); (GK) 27.6.2017 - 17224/11, Rn. 86 f. - Medžlis Islamske Zajednice Brčko and Others v. Bosnia and Herzegovina = AfP 2018, 310 (im Bereich der Religionsausübung); 9.1.2018 - 18597/13, Rn. 57 - GRA Stiftung gegen Rassismus und Antisemitismus v. Switzerland = NJOZ 2019, 493; 8.10.2019 - 15449/09, Rn. 47 - Margulev v. Russia (im Bereich der Stadtplanung). Vgl. auch EGMR, 15.2.2005 - 68416/01, Rn. 89 - Steel and Morris v. UK $=$ NJW 2006, 1255. S. überdies die anhängige Rechtssache 1097/10 - Kashapov v. Russia.

109 EGMR, 17.7.2008 - 42211/07, Rn. 63 - Riolo c. Italie (betr. einen Politikwissenschaftler). Vgl. auch EGMR, 26.5.2009 - 31475/05, Rn. 43 - Kenedi v. Hungary (betr. einen Historiker).

110 EGMR, 29.6.2004 - 64915/01, Rn. 67 f. - Chauvy and Others v. France.

111 Deutlich hinsichtlich des Zugangs zu staatlichen Dokumenten EGMR, 14.4.2009 - 37374/05, Rn. 36 - Társaság a Szabadságjogokért v. Hungary; 28.11.2013 - 39534/07, Rn. 41 ff. - Österreichische Vereinigung zur Erhaltung, Stärkung und Schaffung eines wirtschaftlich gesunden landund forstwirtschaftlichen Grundbesitzes v. Austria; (GK) 8.11.2016 - 18030/11, Rn. 164 ff. - Magyar Helsinki Bizottság v. Hungary = AfP 2017, 301; ähnlich zu vermeintlicher Diffamierung EGMR (GK), 27.6.2017 - 17224/11, Rn. 86 ff. - Medžlis Islamske Zajednice Brčko and Others v. Bosnia and Herzegovina $=$ AfP 2018, 310 .

112 EGMR, 3.4.2012 - 41723/06, Rn. 93, 95 - Gillberg v. Sweden, zum Quellenschutz für Wissenschaftler. 
ten Glauben und unter Einhaltung journalistisch-ethischer Standards akkurate und verlässliche Informationen verbreiten. ${ }^{113}$

Jüngst hat der EGMR darüber hinaus die Bereitschaft erkennen lassen, den Kreis von „watchdogs“ noch einmal zu erweitern. In obiter dicta stellte der Gerichtshof fest, dass die rechtliche Einordnung der Funktion von Bloggern und beliebten Nutzern sozialer Medien (,bloggers and popular users of the social media“) wegen der bereits dargestellten Bedeutung des Internets der von „public watchdogs" angepasst (,assimilated to that of") werden könne. ${ }^{114}$ In dieser Passage kommt eine Sensibilität des Gerichtshofs für den Wandel der Rollenbilder im digitalen Kommunikations- und Informationsprozess zum Ausdruck: Eine Privatperson ist nicht mehr nur passiver Rezipient, sondern - je nach eigenem Wunsch aktiver Nutzer mit eigenem Kontroll- und Wirkpotenzial. Dies ist konsistent mit der andernorts deutlich gewordenen Vorstellung des Gerichtshofs von einer Begünstigung des „citizen journalism“ im Internet ${ }^{115}$. Begrüßenswert ist, dass der EGMR damit eine Offenheit signalisiert, die auch auf internationaler Ebene seit längerem zirkulierenden Überlegungen eines neuen Verständnisses journalistischer Arbeit im Internet ${ }^{116}$ aufzugreifen. Es wird sich jedoch noch zeigen müssen, inwiefern diese Grundidee im Detail praktisch und sinnvoll umsetzbar ist. Bereits die bloß kurzen Ausführungen der EGMR verdeutlichen das Kernproblem dieser Überlegungen: Der betroffene Personenkreis lässt sich - insbesondere bei differenzierender Betrachtung der Übertragbarkeit bestimmter Rechte und Pflichten nur schwer verlässlich definieren. So mutet es beispielsweise wenig plausibel an, die Annahme der Geltung eines Redaktionsgeheimnisses oder besonderer journalistischer Sorgfaltspflichten alleine von der Reichweitenstärke eines Social Media-Auftritts abhängig zu machen. ${ }^{117}$ Genau darauf aber stellte der Gerichtshof in der Rechtssache „Rebechenko v.

113 So bereits EGMR (GK), 8.11.2016 - 18030/11, Rn. 159 - Magyar Helsinki Bizottság v. Hungary = AfP 2017, 301; deutlich EGMR (GK), 27.6.2017 - 17224/11, Rn. 87 f., 107 ff. - Medžlis Islamske Zajednice Brčko and Others v. Bosnia and Herzegovina = AfP 2018, 310. Vgl. auch bereits EGMR, 2.2.2012 - 20240/08, Rn. 33 - Růžový panter, o.s. c. République tchèque.

114 EGMR (GK), 8.11.2016 - 18030/11, Rn. 168 - Magyar Helsinki Bizottság v. Hungary = AfP 2017, 301; aufgegriffen in EGMR, 20.3.2018 - 45791/13, Rn. 57 - Falzon v. Malta, und erstmalig gar als ,general principle“ - in 26.3.2020 - 10090/16, Rn. 87 - Centre for Democracy and the Rule of Law v. Ukraine; s. aus jüngerer Zeit nun auch die konkrete Anwendung in EGMR, 28.8.2018 - 10692/09, Rn. 81 - Savva Terentyev v. Russia.

115 EGMR, 1.12.2015 - 48226/10 und 14027/11, Rn. 52 - Cengiz and Others v. Turkey = NJOZ 2017, 1214, zur besonderen Relevanz von YouTube.

116 S. nur General comment No. 34 des United Nations Human Rights Committee zu Art. 19 ICCPR, abrufbar unter: https://www2.ohchr.org/english/bodies/hrc/docs/gc34.pdf, Rn. 44; Recommendation $\mathrm{CM} / \operatorname{Rec}(2011) 7$ of the Committee of Ministers to member states on a new notion of media, abrufbar unter: https://search.coe.int $/ \mathrm{cm} /$ Pages/result_details.aspx?ObjectID $=09000016805 \mathrm{cc} 2 \mathrm{c0}$; vgl. auch Article 19, The Right to Blog, 2013.

117 S. für Kriterien zur Bestimmung des betroffenen Personenkreises und erste Ansätze differenzierter rechtlicher Behandlung Appendix to Recommendation CM/Rec(2011)7 of the Committee of Ministers to member states on a new notion of media, abrufbar unter: https://search.coe.int $/ \mathrm{cm} / \mathrm{Pa}$ ges/result_details.aspx?ObjectID $=09000016805 \mathrm{cc} 2 \mathrm{c} 0$. 
Russia“ ab, als er das nationale Urteil zulasten eines „Bloggers“ wegen der Reichweite eines Videos auf seinem „YouTube“-Kanal (mehr als 2.000 Abonnenten und mehr als 80.000 Views des Videos) nach den für Journalisten geltenden Maßstäben bewertete. ${ }^{118}$

Die Problematik ist weitaus komplexer und der EGMR bietet bislang noch keine ausreichenden Lösungsansätze an. ${ }^{119}$ Angesichts der Tatsache, dass sich die mit ihr verbundenen Rechtsprobleme in den Konventionsstaaten ${ }^{120}$ zunehmend konkretisieren, ${ }^{121}$ dürfte es jedoch nur noch eine Frage der Zeit sein, bis der EGMR Gelegenheit bekommt, konkretere Kriterien für die Abgrenzung der Rollenbilder zu bestimmen. ${ }^{122}$ Dabei sollte in funktionaler Hinsicht im Vordergrund stehen, ob jemand in einem (objektiv klar erkennbaren) professionell orientierten Selbstverständnis durch kontinuierliche Publikationen zum öffentlichen Diskurs beiträgt. ${ }^{123}$

118 EGMR, 16.4.2019 - 10257/17, Rn. 21, 25, 30 - Rebechenko v. Russia, indes nur in Ausschussbesetzung i.S.v. Art. 26 Abs. 1 EMRK.

119 In EGMR, 29.8.2017 - 16393/14, Rn. 22, 31 f. - Sioutis v. Greece, hat der Gerichtshof zumindest klar das Argument abgelehnt, jeder Nutzer sozialer Medien sei meinungsbildend tätig; vgl. auch EGMR, 21.5.2019 - 42982/08, Rn. 31 - Savelyev v. Russia. Zurückhaltend auch O'Leary, Irish Jurist 2018, 59 (72); für eine kontextsensible Differenzierung zwischen ,high level“ und „,low level“ speech Rowbottom, CLJ 2012, 355 (370 ff.); zur Problematik der Definition von ,Journalimus" Hovlid, Finding a judicial definition of journalism, 2019, S. 209 ff.

120 Ausführlich zum deutschen Recht Schierbaum, Sorgfaltspflichten von professionellen Journalisten und Laienjournalisten im Internet, 2016, passim.

121 S. nur zur aktuellen Frage der Geltung des sog. „Medienprivilegs“ in Art. 85 DSGVO fernab „professioneller Journalisten“ Kahl/Piltz, K\&R 2018, 289 (292ff.); Weberling/Bergann, AfP 2019, 293 (296 f.); Michel, Datenschutzrechtliche Anforderungen an Bloggerinnen und Bürgerjournalistinnen, 2019, S. $131 \mathrm{ff}$; ders., AfP 2019, $490 \mathrm{ff}$. Diesbezüglich zuletzt auch - noch zur Richtlinie 95/46/EG - EuGH, 14.2.2019 - C-345/17, K\&R 2019, 252 (Rn. 55 f.) - Buivids; dazu Rombey, ZD 2019, 301 ff.; Grages/Neben, K\&R 2019, 300 (insb. 303). Vgl. dazu auch EGMR (GK), 27.6.2017 - 931/13, Rn. 175 ff. - Satakunnan Markkinapörssi Oy and Satamedia Oy v. Finland = MR-Int 2019, 6 .

122 Eine Gelegenheit hätte sich dem EGMR wohl in der Rechtssache 58400/14 - Kasprowicz c. Pologne geboten; die Beschwerde wurde indes aufgrund einer einseitigen Erklärung der polnischen Regierung mit Entscheidung v. 24.3.2020 gemäß Art. 37 Abs. 1 Satz 1 lit. c) EMRK aus dem Register gestrichen.

123 Oster, Media Freedom as a Fundamental Right, 2015, S. 62 ff.; ders., Kommunikationsdeliktsrecht, 2019, S. 163 ff. (insb. 171 f.); vgl. auch Brings-Wiesen, in: Spindler/Schuster, § 9c RStV Rn. 17. 
cc) Online-Archive

Anlass für besonders intensive Diskussionen haben in den vergangenen Jahren die OnlineArchive ${ }^{124}$ journalistischer Anbieter geboten. ${ }^{125}$ Diese aktualisieren den klassischen Konflikt zwischen dem Recht auf Achtung des Privatlebens der von Berichterstattungen betroffenen Personen gemäß Art. 8 EMRK ${ }^{126}$ und den Rechten der Anbieter und ihrer Rezipienten auf Verbreitung und Empfang von Informationen gemäß Art. 10 EMRK. Er wird ergänzt um die Aspekte der besseren Auffindbarkeit, leichteren Zugänglichkeit und dauerhaften Verfügbarkeit von persönlichen Daten im Internet. Im Vordergrund steht nicht länger die erstmalige Veröffentlichung und Verbreitung eines Artikels, sondern dessen Verbleib in der öffentlichen Sphäre. ${ }^{127}$ Der EGMR hatte in immerhin drei Entscheidungen Gelegenheit, Maßstäbe zu diesem Themenkomplex zu entwickeln. ${ }^{128}$ „Key case“ ist jedoch die jüngste Rechtssache ,M.L. and W.W. v. Germany“ betreffend mehrere Urteile des Bundesgerichtshofs $(\mathrm{BGH})^{129}$ zur Online-Archivierung der Jahrzehnte zurückliegenden Berichterstattung über den Mord am Schauspieler Walter Sedlmayr unter namentlicher Nennung der Täter (partiell auch unter bildlicher Darstellung). Das Urteil ist nicht nur bemerkenswert, weil es erstmalig substanzielle Ausführungen des EGMR zum Grundkonflikt um die Online-Archive enthielt, ${ }^{130}$ sondern gleichermaßen, weil es - gewiss auch wegen seines direkten Bezugs

124 Vgl. zur Relevanz von Archiven allgemein die Recommendation No. R(2000)13 of the Committee of Ministers to member states on a European policy on access to archives, abrufbar unter: https://rm.coe.int/16804cea4f.

125 S. allein zur Rechtsprechung des BGH die Nachweise bei von Pentz, AfP 2020, 93 (102 ff.). S. zur deutschen Rechtslage umfassend und aus jüngerer Zeit nur Becker, Das Recht auf Vergessenwerden, 2019, passim; Milker, Die Umsetzung des „Rechts auf Vergessenwerden“ im deutschen Recht, 2019, passim.

126 Das BVerfG, 6.11.2019 - 1 BvR 16/13, K\&R 2020, 51 (Rn. 79 ff.) - Recht auf Vergessen I; 25.2.2020 - 1 BvR 1282/17, NJW 2020, 1793 (Rn. 7), hat zuletzt klar zwischen Betroffenheit im allgemeinen Persönlichkeitsrecht und im Recht auf informationelle Selbstbestimmung differenziert.

127 Noch einmal abzugrenzen, ist die Konstellation einer neuen Berichterstattung unter Wiederaufgreifen von alten Informationen, vgl. jüngst BVerfG, 23.6.2020 - 1 BvR 1240/14, juris, Rn. 15.

128 EGMR, 10.3.2009 - 3002/03 und 23676/03 - Times Newspapers Ltd (Nos. 1 and 2) v. UK; 16.7.2013 - 33846/07 - Węgrzynowski and Smolczewski v. Poland = AfP 2014, 517, s. dazu die Kommentare von Mann, K\&R 2013, 553 ff., und Haug, AfP 2014, 503 ff.; EGMR, 28.6.2018 $60798 / 10$ und 65599/10 - M.L. and W.W. v. Germany = NJW 2020, 295, s. dazu den Kommentar von Kröner, K\&R 2018, 544 ff.

129 Konkret ging es um BGH, 15.12.2009 - VI ZR 227/08, BGHZ 183, 353; 15.12.2009 - VI ZR 228/08, juris; 9.2.2010 - VI ZR 243/08, K\&R 2010, 332 ff.; 9.2.2010 - VI ZR 244/08, juris; 20.4.2010 - VI ZR 245/08, NJW 2010, 2728 ff.; 20.4.2010 - VI ZR 246/08, juris.

130 In der Rechtssache „Times Newspapers Ltd (Nos. 1 and 2) v. UK“ ging es um eine Verletzung von Art. 10 EMRK durch eine zeitliche unbegrenzte Haftung der Betreiber von Online-Archiven infolge der sog. ,,internet publication rule“ im britischen Recht. Der EGMR sah in einer unverhältnismäßig langen Haftbarkeit zwar grundsätzlich ein Problem, verneinte im konkreten Fall jedoch wegen der besonderen Umstände eine Verletzung; s. zur Entscheidung auch Heißl, EGMR und Internet, 2012, S. 61 (70 f.). - In der Rechtssache „Wegrzynowski and Smolczewski v. Poland“ 
zur Rechtsprechungspraxis des BGH - jüngst den Grundrechtsdialog mit dem BVerfG befeuert hat ${ }^{131}$.

Die Spezifika des Themenkomplexes lässt der EGMR innerhalb der bereits bekannten Grundsätze der Abwägung von Art. 8 und 10 EMRK zur Geltung kommen: Auf der einen Seite würdigt er den bedeutsamen Beitrag der Online-Archive zur Erhaltung und Bereitstellung von leicht zugänglichen und meist kostenfreien Informationen für die Allgemeinheit und wertet die Archivierung durch Internetmedien ${ }^{132}$ als wertvollen Annex (,valuable secondary role“) zu ihrer Primärfunktion als ,public watchdog“. ${ }^{133}$ Auf der anderen Seite mahnt er wegen der zeitlich unbegrenzten und durch Suchmaschinen verstärkten Auffindbarkeit von Informationen sowie des korrespondierenden Einflusses auf die persönliche Wahrnehmung durch die Öffentlichkeit zu einer nuancierten Begutachtung des gesellschaftlichen Informationsinteresses im Wandel der Zeit. ${ }^{134}$ Insofern hat der Gerichtshof durch sein Urteil in ,M.L. and W.W. v. Germany“ ein solides Fundament für den Umgang mit Online-Archiven geschaffen, aufgrund dessen er im konkreten Fall eine Verletzung des Rechts auf Achtung des Privatlebens verneinte.

Nur ansatzweise geklärt ist bislang indes die gewichtige Frage, welche Schutzvorkehrungen zugunsten der Privatsphäre des Einzelnen und zulasten des Informationsinteresses und der Archivbetreiber noch zumutbar sind. Der EGMR hat lediglich offenbart, gegen welche Lösungswege er opponiert. Grundsätzlich kritisch zeigt er sich gegenüber einer „Neuschreibung der Geschichte“ durch Löschung oder nachträgliche Anonymisierung einer Berichterstattung. ${ }^{135}$ Auch lehnt er wegen der damit potenziell verbundenen „,chilling ef-

wandten sich zwei Anwälte zunächst erfolgreich gegen einen diffamierenden Artikel in der Printausgabe einer polnischen Zeitung, unterließen es jedoch gegen die simultan veröffentlichte Onlineversion vorzugehen. Die Abweisung der Jahre später erhobenen Klage gegen diese Version wegen Verfristung erachtete der EGMR unter den konkreten Umständen nicht als Verletzung von Art. 10 EMRK.

131 BVerfG, 6.11.2019 - 1 BvR 16/13, K\&R 2020, 51 (insb. Rn. 106, 111, 113, 118, 130, 151 f.) Recht auf Vergessen I, s. dazu nur Klass, ZUM 2020, 265 ff.; Ory, AfP 2020, 93 ff. S. jüngst auch BVerfG, 25.2.2020 - 1 BvR 1282/17, NJW 2020, 1793 (Rn. 11 ff.); dazu Mann, K\&R 2020, 434 f. S. zu jüngsten Urteilen des französischen Conseil d'État zum Recht auf Vergessen Reinhardt, DuD 2020, $361 \mathrm{ff}$.

132 Indes dürften sich auch (Privat-)Personen, deren Tätigkeit sich in einer bloßen Archivierung erschöpft, auf Art. 10 EMRK berufen können. S. dazu die anhängigen Rechtssachen 74389/10 und 15503/13 - Pronyakin v. Russia und Kharitonova v. Russia.

133 EGMR, 28.6.2018 - 60798/10 und 65599/10, Rn. 90 - M.L. and W.W. v. Germany = NJW 2020, 295; s. bereits EGMR, 10.3.2009 - 3002/03 und 23676/03, Rn. 27, 45 - Times Newspapers Ltd (Nos. 1 and 2) v. UK; 16.7.2013 - 33846/07, Rn. 59 - Węgrzynowski and Smolczewski v. Poland $=\operatorname{AfP} 2014,517$.

134 EGMR, 28.6.2018 - 60798/10 und 65599/10, Rn. 99 ff. - M.L. and W.W. v. Germany = NJW 2020, 295. S. auch speziell zur zeitsensiblen Verdachtsberichterstattung Regenfus, ZUM 2020, $278 \mathrm{ff}$.

135 EGMR, 16.7.2013 - 33846/07, Rn. 66 - Węgrzynowski and Smolczewski v. Poland = AfP 2014, 517; $28.6 .2018-60798 / 10$ und 65599/10, Rn. $105-$ M.L. and W.W. v. Germany = NJW 2020, 295. S. dazu auch die anhängige Rechtssache 57292/16 - Hurbain c. Belgique. 
fects" nicht nur eine Verpflichtung der Betreiber zur proaktiven Prüfung des eigenen Archivbestands ab, sondern warnt sogar davor, dass bereits die Verpflichtung zur Prüfung nach Anzeige im Einzelfall die Medien wegen des damit verbundenen personellen und zeitlichen Aufwands vor einer identifizierenden Berichterstattung zurückschrecken lassen könnte. ${ }^{136}$

Zuletzt haben sowohl der BGH als auch das BVerfG diese Bedenken zwar aufgegriffen, sodann jedoch als mögliche Zwischenlösung im Interesse eines schonenden Ausgleichs der widerstreitenden Rechtsgüter die Einschränkung der Auffindbarkeit von Inhalten durch Maßnahmen der „Suchmaschinenpessimierung“ ins Spiel gebracht ${ }^{137} .{ }^{138}$ Auf dem Papier klingt dieser Ansatz verlockend. Einzelfragen - insbesondere zur rechtlichen und technischen Realisierbarkeit - sind jedoch noch weitgehend unbeantwortet. Deren Klärung wies das BVerfG den Fachgerichten, der BGH zunächst der Tatsacheninstanz des Kammergerichts Berlin zu. Es bleibt abzuwarten, wie sich diese Rechtsprechungslinie fortentwickeln und ob auch der EGMR Gelegenheit bekommen wird, ihren Grundansatz zu würdigen. Angesichts der allseits betonten Relevanz der auffindbarkeitsverstärkenden Wirkung von Suchmaschinen wird dabei auch von Interesse sein, wie die Gerichte die Pflichtenkreise von Suchmaschinenbetreibern und Inhalteanbietern gegeneinander abgrenzen. ${ }^{139}$

\section{b) Intermediäre}

Die schiere Masse an Informationen und der Hang des Menschen zum kommunikativen Austausch haben das Aufkommen neuer Akteure im digitalen Kommunikations- und Informationsprozess nicht bloß funktionsnotwendig, sondern auch ökonomisch attraktiv gemacht. Systembedingt befinden sich die sog. „Intermediäre“140 in Schlüsselpositionen die-

136 EGMR, 28.6.2018 - 60798/10 und 65599/10, Rn. 103 ff. - M.L. and W.W. v. Germany = NJW 2020, 295; anders hingegen das BVerfG, 6.11.2019 - 1 BvR 16/13, K\&R 2020, 51 (Rn. 119, 138) - Recht auf Vergessen I, das gewisse Anstrengungen und Kosten für ausdrücklich zumutbar hält.

137 BGH, 18.12.2018 - VI ZR 439/17, K\&R 2019, 388 (Rn. 20 ff.), und BVerfG, 6.11.2019 - 1 BvR 16/13, K\&R 2020, 51 (Rn. 117 ff., 128 ff.) - Recht auf Vergessen I, jeweils m. w. Nw. aus der Lit. In diese Richtung bereits HansOLG, 7.7.2015 - 7 U 29/12, K\&R 2015, 668 (670); grundsätzlich befürwortend Höch, K\&R 2015, 632 (633 f.); Brost, AfP 2015, 407 (409); Verweyen/Ruf, MMR 2015, 772 (773f.); Trentmann, MMR 2016, 731 (734f.); dagegen kritisch Feldmann, K\&R 2015, 634 ff. (von dem hier der Begriff der „Suchmaschinenpessimierung“ übernommen ist); Koreng, AfP 2015, 514 (515 ff.); Paal/Hennemann, K\&R 2017, 18 (21).

138 Der EuGH hat dem vergleichbar jüngst - 24.9.2019 - C-136/17, K\&R 2019, 710 (Rn. 76 ff.) GC u.a. - festgestellt, dass Suchmaschinenbetreiber spätestens infolge eines Auslistungsantrags eine Pflicht zur Restrukturierung der Suchergebnisse treffen kann; krit. dazu Meyer/Stakowski, K\&R 2019, 677 (678 f.); Gomille, ZUM 2020, 123 (126 f.).

139 S. zu Suchmaschinen in der Rspr. des EGMR noch unter II. 2. b) bb).

$140 \mathrm{Da}$ „Intermediäre“ an unterschiedlichen Stellen mit entsprechend unterschiedlichen Funktionen in den Kommunikations- und Informationsprozess eingebunden sein können, ist der Begriff zunächst weit zu verstehen. Mit Blick auf die rechtlichen Anforderungen ist indes zwingend zwi- 
ses Prozesses, was Fragen nach ihren Rechten, mehr noch aber nach ihren Pflichten aufwirft.

\section{aa) Öffentliche Kommunikationsräume im Internet}

(Dienste-)Anbieter im Internet stellen den Nutzern verschiedenste Arten öffentlicher Kommunikationsräume zur Verfügung wie Kommentarbereiche, Diskussionsforen, Plattformen für Blogs oder soziale Netzwerke. Deren Funktionsmechanismen - insbesondere das Maß an Gestaltung und Kontrolle dieser Bereiche durch ihre Anbieter ${ }^{141}$ - unterscheiden sich bisweilen erheblich. Gemein ist ihnen jedoch, dass mit den Anbietern (mindestens) eine Entität im Hintergrund die technische Hoheit über den jeweiligen Kommunikationsraum innehat. Diese Machtposition ist verbunden mit der Frage ihrer korrespondierenden Verantwortung, denn ,,with great power comes great responsibility“. 142

Der EGMR konnte sich an der - auch international ${ }^{143}$ und insbesondere auf Ebene der $\mathrm{EU}^{144}$ - intensiv geführten Debatte der Verantwortlichkeit von „Intermediären“ bislang nur ausschnittweise beteiligen. In den relevanten Rechtssachen „Delfi AS v. Estonia“ ${ }^{145}$ „Ma-

schen den verschiedenen Arten von Intermediären, insb. sog. „Hosting-Anbietern“, zu unterscheiden.

141 Wann es sich unter Berücksichtigung einzelner Gestaltungs- und Kontrollelemente um sog. „active intermediaries" handelt sowie ob und inwiefern an sie graduell unterschiedliche Anforderungen zu stellen sind, ist Gegenstand anhaltender Diskussionen.

142 S. für die primär relevante Konstellation sog. „Hosting-Anbieter“ zur deutschen Rechtslage ausführlich Kovacs, Die Haftung der Host-Provider für persönlichkeitsrechtsverletzende Internetäußerungen, 2018, passim; mit besonderem Fokus auf Betreiber sozialer Netzwerke Adelberg, Rechtspflichten und -grenzen der Betreiber sozialer Netzwerke, 2020, passim; mit einem aktuellen Überblick Wagner, GRUR 2020, 329 ff. und 447 ff.

143 S. dazu insb. MacKinnon/Hickock/Bar/Lim, Fostering Freedom Online: The Role of Internet Intermediaries, 2014, passim; Recommendation CM/Rec(2012)4 of the Committee of Ministers to member States on the protection of human rights with regard to social networking services, abrufbar unter: https://go.coe.int/lL7Uv; Recommendation CM/Rec(2018)2 of the Committee of Ministers to member States on the role and responsibilities of internet intermediaries, abrufbar unter: https://go.coe.int/CXCbe; zu offenen Fragen McGonagle, The Council of Europe and Internet Intermediaries: A Case Study of Tentative Posturing, 2019, S. $227 \mathrm{ff}$.

144 S. zur einschlägigen E-Commerce-Richtlinie 2000/31/EG nur Cole/Etteldorf/Ullrich, Cross-Border Dissemination of Online Content, 2020, insb. S. $176 \mathrm{ff}$; dazu im äußerungsrechtlichen Kontext jüngst EuGH, 3.10.2019 - C-18/18, K\&R 2019, 719 (Rn. 46) - Glawischnig-Piesczek. S. auch Communication on Tackling Illegal Content Online - Towards an enhanced responsibility of online platforms, $\operatorname{COM}(2017) 555$ final, und Commission Recommendation of 1.3.2018 on measures to effectively tackle illegal content online, C(2018) 1177 final. Aktuell plant die Europäische Kommission, durch einen „Digital Services Act“ u.a. das Haftungsregime der Richtlinie zu reformieren.

145 EGMR (GK), 16.6.2015 - 64569/09 - Delfi AS v. Estonia = NJW 2015, 2863, insg. krit. - auch zur Signalwirkung des Urteils - bereits die Joint Dissenting Opinion of Judges Sajó and Tsotsoria; dazu Petri, ZD 2015, 469 f.; krit. Brunner, HRLR 2016, 163 ff.; s. auch die vorangehende Kammerentscheidung v. 10.10.2013 = MMR 2014, 35, dazu befürwortend Milstein, MMR 2014, 
gyar Tartalomszolgáltatók Egyesülete and Index.hu Zrt v. Hungary“, ${ }^{146}$ „Pihl v. Sweden“147 und „Høiness v. Norway“148 ging es ausschließlich um rechtswidrige Äußerungen ${ }^{149}$ in Kommentarbereichen bzw. Diskussionsforen auf größeren, kommerziell betriebenen Nachrichtenportalen ${ }^{150}$ oder Websites von gemeinnützigen Organisationen. ${ }^{151}$ Speziell für diese Konstellationen greift der EGMR seit seiner Entscheidung im key case „Delfi AS v. Estonia“" auf vier besondere Parameter zurück, die seine Bewertung der Wechselwirkung von Art. 8 und 10 EMRK im Einzelfall ergänzen ${ }^{152}$ :

- Kontext der Äußerungen: Der Gerichtshof unterschied im Hinblick auf das Umfeld einer Äußerung zwischen großen, professionell verwalteten und kommerziell betriebenen Nachrichtenportalen, die ihre Nutzer bewusst zur Partizipation anregen und in der Folge von einem aktiven Kommentarbereich auch finanziell profitieren, einerseits ${ }^{153}$ und Anbietern, die Inhalte zu anderen Zwecken veröffentlichen, andererseits. ${ }^{154}$ Relevant war für den Gerichtshof auch, ob und in welchem Maße mit den Publikationen auf der jewei-

41; tendenziell auch Haug, AfP 2014, 27 (30); krit. indes Volkmann, K\&R 2013, 762 ff.; Schapiro, ZUM 2014, 201 (203 ff.).

146 EGMR, 2.2.2016 - 22947/13 - Magyar Tartalomszolgáltatók Egyesülete and Index.hu Zrt v. Hungary = NJW 2017, 2091; s. dazu Ziegelmayer, K\&R 2016, 228 f.

147 EGMR, 7.2.2017 - 74742/14 - Pihl v. Sweden.

148 EGMR, 19.3.2019 - 43624/14 - Høiness v. Norway.

149 Die Entscheidungen ergingen in äußerungsrechtlichen Zusammenhängen und lassen sich nicht ohne Weiteres auf Sachverhalte im Bereich des geistigen Eigentums übertragen.

150 Bei denen die Eröffnung von Kommentarbereichen gar „eine journalistische Tätigkeit besonderer Art“ darstellen soll, EGMR, 2.2.2016 - 22947/13, Rn. 79 - Magyar Tartalomszolgáltatók Egyesülete and Index.hu Zrt v. Hungary = NJW 2017, 2091; vgl. auch EGMR (GK), 16.6.2015 64569/09, Rn. 112 f. - Delfi AS v. Estonia $=$ NJW 2015, 2863. S. dazu auch die anhängige Rechtssache 39378/15 - Standard Verlagsgesellschaft mbH v. Austria.

151 S. indes zu einem Bewertungsportal EGMR, 24.11.2015 - 72966/13 - Kucharczyk v. Poland, ohne weitere Problematisierung.

152 EGMR (GK), 16.6.2015 - 64569/09, Rn. 142 ff. - Delfi AS v. Estonia = NJW 2015, 2863; angewandt und fortentwickelt in EGMR, 2.2.2016 - 22947/13, Rn. $60 \mathrm{ff}$. - Magyar Tartalomszolgáltatók Egyesülete and Index.hu Zrt v. Hungary = NJW 2017, 2091; 7.2.2017 - 74742/14, Rn. 28 ff. - Pihl v. Sweden; 19.3.2019 - 43624/14, Rn. 70 ff. - Høiness v. Norway; zul. auch 4.6.2020 - 31955/11, Rn. 53 - Jezior c. Pologne. S. kombinierend Koltay, New Media and Freedom of Expression, 2019, S. $211 \mathrm{ff}$.

153 Judges Sajó and Tsotsoria kritisieren in ihrer Joint Dissenting Opinion zu EGMR (GK), 16.6.2015 - 64569/09 - Delfi AS v. Estonia, Rn. 1, 16, 27 ff., 39, eine Geringschätzung des journalistischen Kontexts der Tätigkeit dieser „active intermediaries“ durch die Mehrheit; in diese Richtung auch bereits Schapiro, ZUM 2014, 201 (203 f.).

154 Vgl. EGMR (GK), 16.6.2015 - 64569/09, Rn. 144 - Delfi AS v. Estonia = NJW 2015, 2863, gegenüber 2.2.2016 - 22947/13, Rn. 64, 73 - Magyar Tartalomszolgáltatók Egyesülete and Index.hu Zrt v. Hungary = NJW 2017, 2091 und 7.2.2017 - 74742/14, Rn. $31-$ Pihl v. Sweden, wo (auch) gemeinnützige Organisation betroffen waren, sowie jüngst 4.6.2020 - 31955/11, Rn. 55 Jezior c. Pologne, wo es um den Blog eines Privatmannes (betrieben in seiner Funktion als Kandidat für eine kommunale Wahl) ging. 
ligen Website ein konkreter Anlass für Kommentare gegeben wurde und insbesondere beleidigende Äußerungen provoziert wurden. ${ }^{155}$ Weiterhin war in „Høiness v. Norway“ von Interesse, ob es sich um einen räumlich unmittelbar mit der Publikation verbundenen Kommentarbereich oder ein allgemeines Diskussionsforum des Betreibers handelte. ${ }^{156}$

- Haftung des Urhebers der Äußerung: Darüber hinaus verlangte der EGMR eine Prüfung der rechtlichen wie praktischen Durchsetzbarkeit von Ansprüchen gegenüber den primär verantwortlichen Personen. ${ }^{157}$ Dabei berücksichtigte er das grundsätzlich berechtigte Anonymitätsinteresse der Nutzer ${ }^{158}$ und erkannte an, dass ihre Identifizierung alleine anhand einer IP-Adresse nur selten erfolgsversprechend sein dürfte ${ }^{159}$. Für den Fall, dass ein Anbieter dem nicht durch einen begrenzten Nachweis oder gar eine sichere Authentifizierung der Identität im Vorfeld begegnet, hielt der EGMR dessen Haftung für vertretbar. ${ }^{160}$ Ergänzend ging der Gerichtshof in jüngerer Zeit auf die Problematik der (fortwährenden) Auffindbarkeit rechtswidriger Äußerungen über Suchmaschinen ein und betonte - unter Rekurs auf die Rechtsprechung des EuGH - die diesen gegenüber bestehende Möglichkeit der Geltendmachung eigener Auslistungsansprüche. ${ }^{161}$

- Schutzvorkehrungen der Betreiber: Dem Umstand, welche Art von Schutzvorkehrungen die Betreiber im Hinblick auf die Verbreitung von Inhalten Dritter vorgesehen hatten, maß der EGMR besondere Bedeutung bei. Dabei unterschied er zwischen präventiven und reaktiven Maßnahmen. Die präventiven Maßnahmen der Aufnahme eines Disclai-

155 EGMR, 2.2.2016 - 22947/13, Rn. 72 - Magyar Tartalomszolgáltatók Egyesülete and Index.hu Zrt v. Hungary $=$ NJW 2017, 2091; 4.6.2020 - 31955/11, Rn. 55 - Jezior c. Pologne; s. indes (GK) 16.6.2015 - 64569/09, Rn. 144 - Delfi AS v. Estonia = NJW 2015, 2863, wo der Gerichtshof darauf hinwies, dass auch ausgewogene Artikel zu (vermeintlich) neutralen Themen im Internet heftige Diskussionen provozieren mögen; s. auch EGMR, 7.2.2017 - 74742/14, Rn. 30 - Pihl v. Sweden, wo er es für relevant erachtete, dass ein kontextunabhängiger Kommentar nicht vorhersehbar war.

156 EGMR, 19.3.2019 - 43624/14, Rn. 72 - Høiness v. Norway.

157 Besonders deutlich jüngst EGMR, 4.6.2020 - 31955/11, Rn. 59 - Jezior c. Pologne; s. dazu auch EGMR, 24.11.2015 - 72966/13, Rn. 38 ff. - Kucharczyk v. Poland; vgl. bereits EGMR, 2.12.2008 - 2872/02 - K.U. v. Finland.

158 EGMR (GK), 16.6.2015 - 64569/09, Rn. 147 ff. - Delfi AS v. Estonia = NJW 2015, 2863; s. auch EGMR, 24.4.2018 - 62357/14, Rn. 105 f., 117 - Benedik v. Slovenia.

159 EGMR (GK), 16.6.2015 - 64569/09, Rn. 150 - Delfi AS v. Estonia = NJW 2015, 2863; krit. dazu Brunner, HRLR 2016, 163 (172 f.).

160 EGMR (GK), 16.6.2015 - 64569/09, Rn. 151 - Delfi AS v. Estonia = NJW 2015, 2863; noch schärfer die Concurring Opinion of Judge Zupančič. Für problematisch erachtete der EGMR indes das Fehlen jeglicher Überlegungen zu dieser Frage, 2.2.2016 - 22947/13, Rn. 78 - Magyar Tartalomszolgáltatók Egyesülete and Index.hu Zrt v. Hungary = NJW 2017, 2091; dies sei nicht mit der Rechtsprechung zur Sanktionierung von Journalisten für das Verbreiten von Äußerungen Dritter vereinbar, a.a.O., Rn. 79. Demgegenüber wertete er jüngst, 4.6.2020 - 31955/11, Rn. 57 Jezior c. Pologne, die kurzzeitige Einführung der Verpflichtung zur Angabe einer E-Mail-Adresse zugunsten des Betreibers.

161 EGMR, 7.2.2017 - 74742/14, Rn. 33 - Pihl v. Sweden. S. dazu auch noch unter II. 2. b) bb). 
mers oder eines Verbots bestimmter Äußerungen (in die allgemeinen Geschäftsbedingungen $)^{162}$ bewertete der Gerichtshof positiv, wohl aber nicht als ausreichend. In der Rechtssache „Delfi AS v. Estonia“ hielt er den konkret verwendeten automatisierten Wortfilter wegen seiner fehlenden Sprach- und Kontextsensibilität für ungeeignet. ${ }^{163}$ Demgegenüber erachtete er in der Rechtssache „Magyar Tartalomszolgáltatók Egyesülete and Index.hu Zrt v. Hungary" die Annahme, dass bereits die Möglichkeit des ungefilterten Kommentierens die Verantwortlichkeit der Websitebetreiber begründen solle, als unverhältnismäßig, nicht praktikabel und geeignet, die Informationsfreiheit im Internet $\mathrm{zu}$ unterminieren. ${ }^{164}$ Im Hinblick auf reaktive Maßnahmen gestand der EGMR zu, dass ein ,notice and take down"-Verfahren, das innerhalb eines effektiven Mechanismus schnelle Reaktionen ermöglicht, in vielen Fällen als geeignetes Instrument zum Ausgleich der widerstreitenden Interessen dienen kann. ${ }^{165}$ Zugleich erachtete er es jedoch in Fällen von eindeutig rechtswidrigen Äußerungen, wie Hassrede oder unmittelbaren Gewaltandrohungen, ${ }^{166}$ als mit Art. 10 EMRK vereinbar, wenn Mitgliedstaaten von Websitebetreibern die unverzügliche Löschung dieser Kommentare auch ohne vorherigen

162 EGMR (GK), 16.6.2015 - 64569/09, Rn. 155 f. - Delfi AS v. Estonia = NJW 2015, 2863; 2.2.2016 - 22947/13, Rn. 81 - Magyar Tartalomszolgáltatók Egyesülete and Index.hu Zrt v. Hungary $=$ NJW 2017, 2091; vgl. auch EGMR, 4.6.2020 - 31955/11, Rn. 56 - Jezior c. Pologne.

163 EGMR (GK), 16.6.2015 - 64569/09, Rn. 156 - Delfi AS v. Estonia = NJW 2015, 2863; dabei ließ er die Verhältnismäßigkeit des Einsatzes von Filtertechnik im Übrigen offen. In der Tendenz anders jüngst der EuGH, 3.10.2019 - C-18/18, K\&R 2019, 719 (Rn. 46) - Glawischnig-Piesczek, krit. dazu Spindler, NJW 2019, 3274 (3275); Heldt, EuR 2020, 238 (243 f.); Keller, GRUR Int. 2020, 616 ff.; differenzierend Holznagel, ZUM 2019, 910 ff. Vgl. aber zu Filtersystemen im urheberrechtlichen Kontext EuGH, 16.2.2012 - C-360/10, GRUR 2012, 382 (Rn. 39 ff.) - SABAM; auch 24.11.2011 - C-70/10, MMR 2012, 174 (Rn. 41 ff.) - Scarlet Extended.

164 EGMR, 2.2.2016 - 22947/13, Rn. 82 - Magyar Tartalomszolgáltatók Egyesülete and Index.hu Zrt v. Hungary $=$ NJW 2017, 2091; aufgegriffen in 7.2.2017 - 74742/14, Rn. $31-$ Pihl v. Sweden; 4.6.2020 - 31955/11, Rn. 56 - Jezior c. Pologne; in diese Richtung bereits die Joint Concurring Opinion of Judges Raimondi, Karakaş, De Gaetano and Kjølbro zu (GK), 16.6.2015 - 64569/09 - Delfi AS v. Estonia, Rn. 6 f.

165 S. für einen differenzierten Ansatz je nach Kontext Angelopoulos/Smet, JML 2016, 266 (275 ff.).

166 Vgl. die unterschiedliche Handhabung in EGMR (GK), 16.6.2015 - 64569/09, Rn. 140 - Delfi AS v. Estonia = NJW 2015, 2863, sowie 19.3.2019 - 43624/14, Rn. 69, 71 - Høiness v. Norway, gegenüber EGMR, 2.2.2016 - 22947/13, Rn. 64, 70, 74 ff. - Magyar Tartalomszolgáltatók Egyesülete and Index.hu Zrt v. Hungary = NJW 2017, 2091, sowie 7.2.2017 - 74742/14, Rn. $25-$ Pihl v. Sweden. Krit. zu dieser Differenzierung Angelopoulos, JIPLP 2016, 582 (584). 
Hinweis ${ }^{167}$ verlangen. ${ }^{168}$ Konkretere Erwartungen an Art und Maß der Moderation von Kommentaren hat der EGMR bislang zurückgehalten. ${ }^{169}$

- Konsequenzen für die Websitebetreiber: Abschließend bezog der Gerichtshof die Konsequenzen der Haftbarkeit der Websitebetreiber in die Bewertung ein. Dabei blickte er aus der Vogelperspektive auf die im nationalen Rechtssystem geltenden Maßstäbe der Verantwortlichkeit und prüfte deren möglichen ,chilling effect“ auf die Gestaltung der Kommunikationsräume, wobei er eine besonders intensive Wirkung auf Betreiber nicht kommerzieller Websites erkannte. ${ }^{170}$

Der EGMR hat bislang stets die Spezifika dieser Fälle betont ${ }^{171}$ und insbesondere in „Delfi $A S$ v. Estonia“ gemahnt, die neu etablierten Maßstäbe nicht ohne Weiteres auf andere Kom-

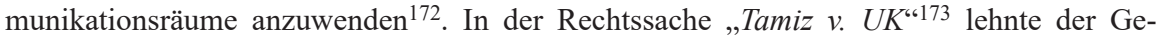
richtshof daher eine Berufung auf die dargestellten Erwägungen der Verantwortlichkeit in

167 Somit erteilt der EGMR einer gemäß Art. 15 E-Commerce-Richtlinie und § 7 Abs. 2 TMG verbotenen allgemeinen Überwachungspflicht de facto das Siegel der Konventionskonformität, so bereits Brings-Wiesen, Das Phänomen der „Online Hate Speech“ aus juristischer Perspektive, 2017, S. 35 (39); auch Petri, ZD 2015, 469 (470); sehr krit. dazu bereits die Joint Dissenting Opinion of Judges Sajó and Tsotsoria zu EGMR (GK), 16.6.2015 - 64569/09 - Delfi AS v. Estonia, Rn. 33 ff.; krit. zur fehlenden substanziellen Berücksichtigung der Wertungen der E-CommerceRichtlinie und der einschlägigen Rspr. des EuGH darin Brunner, HRLR 2016, 163 (167 ff.); auch Husovec, JIPLP 2016, 17 (20).

168 EGMR (GK), 16.6.2015 - 64569/09, Rn. 159 - Delfi AS v. Estonia = NJW 2015, 2863; bestätigt in EGMR, 2.2.2016 - 22947/13, Rn. 91 - Magyar Tartalomszolgáltatók Egyesülete and Index.hu Zrt v. Hungary $=$ NJW 2017, 2091; vgl. auch EGMR, 7.2.2017 - 74742/14, Rn. 32, $36-$ Pihl v. Sweden; 30.1.2018 - 59040/08, Rn. 38 f. - Oktar c. Turquie.

169 Bislang hat der EGMR eine (auch nur sporadische) Moderation weitgehend unkommentiert, indes tendenziell positiv zur Kenntnis genommen, vgl. EGMR (GK), 16.6.2015 - 64569/09, Rn. 157 - Delfi AS v. Estonia = NJW 2015, 2863; 2.2.2016 - 22947/13, Rn. 81 - Magyar Tartalomszolgáltatók Egyesülete and Index.hu Zrt v. Hungary = NJW 2017, 2091; 19.3.2019 43624/14, Rn. 72 - Høiness v. Norway; 4.6.2020 - 31955/11, Rn. 56 f. - Jezior c. Pologne; s. demgegenüber die Joint Concurring Opinion of Judges Raimondi, Karakaş, De Gaetano and Kjølbro zu (GK), 16.6.2015 - 64569/09 - Delfi AS v. Estonia, Rn. 9 ff.

170 EGMR, 2.2.2016 - 22947/13, Rn. 86 - Magyar Tartalomszolgáltatók Egyesülete and Index.hu Zrt v. Hungary $=$ NJW 2017, 2091; 7.2.2017 - 74742/14, Rn. $35-$ Pihl v. Sweden; 4.6.2020 31955/11, Rn. 60 - Jezior c. Pologne; vgl. demgegenüber die höheren Anforderungen in EGMR (GK), 16.6.2015 - 64569/09, Rn. 160 f. - Delfi AS v. Estonia = NJW 2015, 2863. S. zur Berücksichtigung des ,chilling effect“" näher Fathaigh, EHRLR 2017, 387 ff.

171 Grundlegend EGMR (GK), 16.6.2015 - 64569/09, Rn. 111 ff. - Delfi AS v. Estonia = NJW 2015, 2863; vgl. dazu bewusst unterscheidend EGMR, 2.2.2016 - 22947/13, Rn. 64, 70 - Magyar Tartalomszolgáltatók Egyesülete and Index.hu Zrt v. Hungary = NJW 2017, 2091; 7.2.2017 74742/14, Rn. 31 - Pihl v. Sweden; 19.3.2019 - 43624/14, Rn. 69, 71 - Høiness v. Norway; auch Spano, HRLR 2017, 665 (675 ff.) bezweifelt daher die präzedenzielle Wirkung des Urteils der GK in Delfi AS v. Estonia; in diese Richtung auch Oster, European and International Media Law, 2017, S. $240 \mathrm{f}$.

172 EGMR (GK), 16.6.2015 - 64569/09, Rn. 116 - Delfi AS v. Estonia = NJW 2015, 2863.

173 EGMR, 19.9.2017 - 3877/14, Rn. 80 ff. - Tamiz v. UK. 
einem Verfahren gegen die „Google Inc.“ als Anbieterin des Blog Hosting-Dienstes „Blogger.com" ab. Erst jüngst deutete sich in der Entscheidung der Rechtssache „Jezior c. Pologne $e^{\text {174 }}$ an, dass er auch Konstellationen der Verantwortlichkeit von Privatpersonen für Kommentarbereiche auf ihren Blogs graduell anders bewerten will. Es bleibt daher abzuwarten, ob und inwieweit die genannten Parameter ${ }^{175}$ auf Kommunikationsräume mit anderen Funktionsmechanismen wie insbesondere soziale Netzwerke ${ }^{176}$ übertragen werden. Die aktuell anhängigen Rechtssachen „Sanchez c. France“177 und „Glukhov v. Russia“"178 werden dem EGMR zunächst Gelegenheiten bieten, Fragen zur Verantwortlichkeit der Betreiber von „Facebook“-Profilen für Inhalte Dritter zu klären. Angesichts der vielerorts vernehmbaren Tendenz zur gezielteren Inpflichtnahme von Intermediären ${ }^{179}$ bleibt jedoch zu hoffen, dass der Gerichtshof auch Gelegenheit bekommen wird, für deren - durch konventionsstaatliche Normen flankierten - Kontrollsysteme rechtsstaatliche Grundsätze zu konkretisieren. Die jüngsten Ansätze des „Flaggings“ von oder der Gegendarstellung zu problematischen Inhalten ${ }^{180}$ beweisen einmal mehr, dass letztlich Details der Moderationspra-

174 EGMR, 4.6.2020 - 31955/11, Rn. 55, 60 - Jezior c. Pologne, indes nur in Ausschussbesetzung i.S.v. Art. 26 Abs. 1 EMRK.

175 Abzuwarten bleibt auch, in welchem Maße der Gerichtshof von den Diensteanbietern zukünftig eine Wahrung der Rechte der Urheber von Äußerungen, insbesondere ein Recht auf Anhörung und „put back“, verlangt. S. zur Diskussion um diese Rechtspositionen Holznagel, CR 2019, $518 \mathrm{ff}$. Aktuell avisiert die Bundesregierung auch die Etablierung eines sog. „Gegenvorstellungsverfahrens" im NetzDG, BT-Drs. 19/18792, S. 8 f., 46 f.

176 So bspw. hinsichtlich der Löschung wort- und sinngleicher Inhalte auf Facebook wie in EuGH, 3.10.2019 - C-18/18, K\&R 2019, 719 - Glawischnig-Piesczek.

177 45581/15 - Sanchez c. France (betr. das Profil eines Bürgermeisters und Kandidaten für eine Parlamentswahl).

178 42633/18 - Glukhov v. Russia (betr. das Profil eines Anwalts).

179 Das deutsche NetzDG dürfte hier eine wesentliche Vorreiterrolle gespielt haben; zu dessen Vereinbarkeit mit Art. 10 EMRK MacMillan, CJIL 2019, 252 ff.; mit Art. 19 IPbpR Zipursky, FILJ 2019, 1325 ff. Die EU hat dessen Grundideen bereits mehrfach verschiedentlich in ihrer Sekundärrecht einfließen lassen, vgl. nur Art. 1 Nr. 23 der Richtlinie (EU) 2018/1808, ABl. EU v. 28.11.2018, Nr. L 303, S. 69 (87 ff.), sowie Art. 17 der Richtlinie (EU) 2019/790, ABl. EU v. 17.5.2019, Nr. L 130, S. 92 (119ff.); vgl. auch den Vorschlag für eine Verordnung zur Verhinderung der Verbreitung terroristischer Online-Inhalte, $\operatorname{COM}(2018) 640$ final; auch der bereits erwähnte „Digital Services Act“ dürfte in seiner Reform der Vorgaben der E-Commerce-Richtlinie die Anforderungen an Intermediäre intensivieren. Jüngst hat der Conseil Constitutionel, 18.6.2020 - 2020-801 DC, das frz. Pendant zum NetzDG für teilweise verfassungswidrig erklärt; dazu Heldt, JuWissBlog Nr. 96/2020.

180 S. dazu nur die letzten Änderungen von Twitter betr. ,misleading information“ in Reaktion auf die COVID-19-Pandemie Roth/Pickels, Twitter-Blog v. 11.5.2020. 
xis $^{181}$ - auch unter Ausschöpfung technischer Möglichkeiten ${ }^{182}$ - im Fokus der Betrachtung einer ausbalancierten Regulierung öffentlicher Kommunikationsräume im globalen ${ }^{183}$ Internet stehen. ${ }^{184}$

\section{bb) Suchmaschinen}

Nicht minder interessant sind Rolle und Funktion von Suchmaschinen: Sie tragen nicht nur wesentlich zur Auffindbarkeit von Informationen bei, sondern können durch individualisierte Gewichtung auch erheblich deren Wahrnehmbarkeit beeinflussen. ${ }^{185}$

Dem EGMR bot sich bislang kein Anlass, deren Wirken unmittelbar am Maßstab von Art. 10 EMRK zu bewerten. Erste verwertbare Erwägungen lassen sich der bereits erwähnten Entscheidung in der Rechtssache „M.L. and W.W. v. Germany“ entnehmen. Quasi obiter dictum stellte der Gerichtshof fest, dass die Verantwortlichkeit für persönlichkeitsrechtsbeeinträchtigende Äußerungen zwar originär bei den Urhebern liege, Suchmaschinen jedoch deren Wirkung verstärkten. Aus eben diesem Effekt leitet der EGMR eine Verantwortlichkeit auch von Suchmaschinenbetreibern ab, erkennt jedoch zugleich an, dass sich deren Pflichten von Inhalteanbietern unterscheiden mögen. ${ }^{186}$ Bemerkenswert ist, wie deutlich der EGMR - unter Rekurs auf die Rechtsprechung des EuGH ${ }^{187}$ - den Wertungsunterschied zwischen Betreibern von Suchmaschinen und von journalistischen Archiven auf deren divergierende Funktion und Intention zurückführt. Vor diesem Hintergrund verwundert nicht,

181 Wobei sich mit zunehmender Moderation auch die Frage der Kontrolle von und somit der Verantwortlichkeit für fremde Inhalte aktualisieren würde, s. dazu nur Oster, Kommunikationsdeliktsrecht, 2019, S. $350 \mathrm{ff}$.

182 Mit dem Einsatz von Algorithmen oder künstlicher Intelligenz zur Moderation von Inhalten hat sich der EGMR noch nicht befasst, s. dazu aber die Aktivitäten des Europarats, abrufbar unter: https://www.coe.int/en/web/freedom-expression/algorithms-and-human-rights; insb. die Declaration Decl(13/02/2019)1 by the Committee of Ministers on the manipulative capabilities of algorithmic processes, abrufbar unter: https://go.coe.int/oXzdw, sowie die Recommendation CM/ $\operatorname{Rec}(2020) 1$ of the Committee of Ministers to member States on the human rights impacts of algorithmic systems, abrufbar unter: https://go.coe.int/lBwbG.

183 Auch die Frage der geographischen Reichweite einer notwendigen Moderation persönlichkeitsrechtsverletzender Inhalte ist bislang offengeblieben, s. zul. auf die nationalen Rechtsordnungen verweisend EuGH, 3.10.2019 - C-18/18, K\&R 2019, 719, Rn. 48 ff. - Glawischnig-Piesczek; krit. dazu Spindler, NJW 2019, 3274 (3276f.).

184 S. ähnlich auch noch zu Suchmaschinen unter II. 2. b) bb).

185 S. dazu die Recommendation CM/Rec(2012)3 of the Committee of Ministers to member States on the protection of human rights with regard to search engines, abrufbar unter: https://go.coe.int/ bbdcx; auch Koltay, New Media and Freedom of Expression, 2019, S. $115 \mathrm{ff}$.

186 EGMR, 28.6.2018 - 60798/10 und 65599/10, Rn.91, 97 - M.L. and W.W. v. Germany = NJW 2020, 295; entsprechend unterscheidend auch BVerfG, 6.11.2019 - 1 BvR 276/17, K\&R 2020, 59 (Rn. 112 ff.) - Recht auf Vergessen II.

187 EuGH, 13.5.2014 - C-131/12, K\&R 2014, 502 ff. - Google Spain und Google; s. nun auch EuGH, 24.9.2019 - C-136/17, K\&R 2019, 710 ff. - GC u.a., sowie 24.9.2019 - C-507/17, K\&R 2019, 716 ff. - Google. 
dass der EGMR auch für relevant erachtete, ob Betroffene ihre Auslistungsansprüche bereits gegen Suchmaschinenbetreiber gerichtet hatten. ${ }^{188}$ Angesichts des Zusammenspiels und der Wechselwirkung zwischen Websites und Suchmaschinen verbietet sich jedoch eine starre Hierarchie der Anspruchsgegner; im Einzelfall sind die konfligierenden Interessen aller Beteiligten gegeneinander abzuwägen. ${ }^{189}$ Eine weitere Klärung dieses Verhältnisses könnte durch die anhängige Rechtssache „Hurbain c. Belgique“ erfolgen. ${ }^{190}$

Bei der weiteren Konkretisierung seiner Rechtsprechung sollte der EGMR gleichsam darauf bedacht sein, die Maßstäbe für Suchmaschinen funktionsorientiert zu bestimmen und klar von jenen für andere „Intermediäre“ abzugrenzen. ${ }^{191}$

\section{Welche Entwicklungen zeigen sich angesichts eines sich wandelnden Kommunikationsmodus?}

Zuletzt ist zu berücksichtigen, dass das Internet verändert, wie wir miteinander kommunizieren. Der digitale Raum bietet nicht bloß bislang unbekannte Kommunikationsformen, sondern schafft auch eine neue Kommunikationsatmosphäre.

\section{a) Internetspezifische Formen der Kommunikation}

Angesichts seiner Offenheit für die verschiedensten Formen der Kommunikation ${ }^{192}$ ist mit Spannung zu erwarten, wie der EGMR deren vielfältige Facetten im Internet würdigt. Gelegenheit für eine substanzielle Positionierung bot sich dem Gerichtshof bislang nur zu einem der praktisch relevantesten Phänomene - dem „Hyperlinking“. 193

In der Rechtssache „Magyar Jeti Zrt v. Hungary“ - betreffend Verlinkungen auf einem Nachrichtenportal - erkannte der EGMR an, dass Hyperlinks durch ihre Navigationsfunktion zum reibungslosen Funktionieren des Internets beitragen und dass bestimmte Differen-

188 EGMR, 28.6.2018 - 60798/10 und 65599/10, Rn. 114 - M.L. and W.W. v. Germany = NJW 2020, 295; so bereits EGMR, 19.10.2017 - 71233/13, Rn. 52 f. - Fuchsmann v. Germany = NJW 2018, 3083; vgl. auch EGMR, 7.2.2017 - 74742/14, Rn. 33 - Pihl v. Sweden.

189 Vgl. BVerfG, 6.11.2019 - 1 BvR 276/17, K\&R 2020, 59 (Rn. 112, 115 ff.) - Recht auf Vergessen II. In diese Richtung auch EuGH, 13.5.2014 - C-131/12, K\&R 2014, 502 (Rn. 84) - Google Spain und Google; 24.9.2019 - C-136/17, K\&R 2019, 710 (Rn. 52) - GC u.a.; 24.9.2019 C-507/17, K\&R 2019, 716 (Rn. 44) - Google, und BGH, 27.2.2018 - VI ZR 489/16, BGHZ 217, 350 (Rn. 45, 51); vgl. jüngst OLG Karlsruhe, 10.6.2020 - 6 U 129/18, juris, Rn. 78-80.

190 57292/16 - Hurbain c. Belgique.

191 Vgl. hinsichtlich der Störerhaftung nach deutschem Recht bereits treffend BGH, 27.2.2018 - VI ZR 489/16, BGHZ 217, 350 (Rn. 33 ff.).

192 Brings-Wiesen, in: Spindler/Schuster, Erster Teil. A. Rn. 24 f.

193 Fragen der Haftung für Hyperlinks haben sich auf deutscher und EU-Ebene bislang weniger im Äußerungs- und mehr im Recht des geistigen Eigentums gestellt, s. dazu nur in: Heckmann, jurisPK-Internetrecht, Heckmann/Paschke, Kap. 3.1 (Stand: 18.5.2020), insb. Rn. 19, 179, 204 ff., 581, sowie Roggenkamp/Stadler, Kap. 10 (Stand: 16.04.2019), Rn. 418 ff. 
zierungsmerkmale die Bewertung ihres Einsatzes leiten ${ }^{194}$ : So seien sie grundsätzlich nicht mit traditionellen Methoden der journalistischen Berichterstattung vergleichbar, da sie die Rezipienten nur auf die Existenz eines andernorts verfügbaren Inhalts aufmerksam machten, ohne diesen jedoch selbst wiederzugeben. ${ }^{195}$ Über diesen Inhalt habe der Verlinkende im Normalfall auch keine Kontrolle: Er sei auch ohne den Hyperlink frei verfügbar und könne nachträglich beliebig modifiziert werden. Es ist unübersehbar, dass der EGMR damit die - ausdrücklich als „relevant international law and practice“ identifizierte ${ }^{196}$ - Rechtsprechung des EuGH ${ }^{197}$ aufgreift. Dabei gelingt es ihm jedoch im Weiteren, diese vom urheberrechtlichen in den äußerungsrechtlichen Kontext zu übertragen ${ }^{198}$ : Das bloße Setzen eines Hyperlinks könne nicht pauschal als Verbreitung darüber erreichbarer diffamierender Informationen erachtet werden. Vielmehr bedürfe es einer Bewertung unter Berücksichtigung der Besonderheiten des Einzelfalls. Streitgegenstand der besagten Rechtssache war ein Artikel auf einem professionell betriebenen Nachrichtenportal. Dementsprechend hielt der EGMR für relevant, ob der Autor die diffamierende Äußerung befürwortet, wiederholt oder lediglich auf sie verlinkt hatte und ob er von ihrer Rechtswidrigkeit wusste oder hätte wissen können und insofern unter Einhaltung journalistischer Sorgfaltspflichten gearbeitet hatte. 199

194 S. zu allem EGMR, 4.12.2018 - 11257/16, Rn. 73 ff. - Magyar Jeti Zrt v. Hungary = NJW 2019, 3201; vgl. auch bereits die sehr weitgehende Berücksichtigung von Hyperlinks in EGMR (GK), 13.7.2012 - 16354/06, Rn. 73-75 - Mouvement raëlien suisse v. Switzerland.

195 Der EGMR, s. nur 10.7.2014 - 48311/10, Rn. 69 f. - Axel Springer AG v. Germany (no. 2) = NJW 2015, 1501, geht allgemein davon aus, dass Journalisten bei der Wiedergabe von Äußerungen Dritter im Regelfall keine Prüfpflicht trifft.

196 EGMR, 4.12.2018 - 11257/16, Rn. 29 - Magyar Jeti Zrt v. Hungary = NJW 2019, 3201.

197 Konkret EuGH, 8.9.2016 - C-160/15, K\&R 2016, 661 (Rn. 39 ff.) - GS Media. Fortentwickelt in EuGH, 26.4.2017 - C-527/15, K\&R 2017, 396 (Rn. 23 ff.) - Stichting Brein; 7.8.2018 C-161/17, K\&R 2018, 562 (Rn. 39 ff.) - Renckhoff; 29.7.2019 - C-516/17, K\&R 2019, 574 (Rn. 75 ff.) - Spiegel Online; s. auch bereits EuGH, 13.2.2014 - C-466/12, K\&R 2014, 256 (Rn. 14 ff.) - Svensson u.a. S. zum Dialog der Gerichte in diesem Sachkomplex Oręziak, ICLR 2019, $432 \mathrm{ff}$.

198 S. zu allem EGMR, 4.12.2018 - 11257/16, Rn. 76 ff. - Magyar Jeti Zrt v. Hungary = NJW 2019, 3201.

$199 \mathrm{Zu}$ begrüßen ist insofern der Rekurs auf die Rechtsprechung zur Wiedergabe von Äußerungen Dritter, ausgehend von EGMR (GK), 23.9.1994 - 15890/89, Rn. 35 - Jersild v. Denmark = NStZ 1995, 237; zuletzt EGMR, 8.10.2019 - 29097/08, Rn. 48 - Nadtoka v. Russia (No. 2). Vgl. dazu auch die anhängige Rechtssache 77940/17 - Index.hu Zrt v. Hungary. S. zum journalistischen Gebrauch auf nationaler Ebene auch bereits BGH, 14.10.2010 - I ZR 191/08, BGHZ 187, 240 ff. - AnyDVD, krit. dazu Barnitzke, K\&R 2011, 329 ff.; akzeptierend BVerfG, 15.12.2011 1 BvR 1248/11, BVerfGK 19, 278 ff.; dazu auch Mann/Smid, in: Spindler/Schuster, Siebter Teil. Rn. $70 \mathrm{ff}$. 
Diese Leitlinien dürften - insbesondere hinsichtlich des Pflichtenkanons der Verlinkenden - durch zukünftige Entscheidungen weiter ausdifferenziert werden. ${ }^{200}$ Aktuell anhängige Rechtssachen lassen darüber hinaus erwarten, dass der Gerichtshof zeitnah auch zu den Kommunikationsspezifika des ,,(Re-)Postens" und „Sharens“201 oder „Likens“"202 in sozialen Netzwerken wie „Facebook“ oder „VKontakte“ erste Bewertungsmaßstäbe formulieren wird. ${ }^{203}$

\section{b) „Der gute Ton “ im Internet}

In ständiger Rechtsprechung spricht der EGMR nicht nur dem gewählten Ausdrucksstil und damit auch einem besonders scharfen Ton - einen spezifischen Schutz unter Art. 10 EMRK zu, ${ }^{204}$ er unternimmt überdies auch eine kontextabhängige Prüfung von Äußerungen $^{205}$. Vor diesem Hintergrund ist es nur konsequent, auch mögliche Besonderheiten des Kommunikationsstils im Internet zu berücksichtigen. ${ }^{206}$ Sofern ein niedriges Niveau dem geläufigen Kommunikationsstil entspricht, soll erwogen werden, dass dies die Wirkung diffamierender Äußerungen verringern kann.

Dass der EGMR eine Spezifik der Kommunikationsatmosphäre im Internet wahrnimmt und als relevante Größe in die Dogmatik von Art. 10 EMRK inkorporiert, ist grundsätzlich

200 S. die Rechtssachen: 8921/15, 10964/15, 14673/17 und 30328/17 - Garapova, Aronson, Zavolgin und Bondar v. Russia; 40050/17 und 50535/17 - OOO Redaktsiya 7x7 and Yezhova v. Russia; 9578/19 u.a. - OOO Redaktsiya 7x7 and Yezhova v. Russia.

201 S. die anhängigen Rechtssachen betr. Facebook: 14307/17 - Gahraman v. Azerbaijan; 44652/18 Ponta c. Roumaine; 74009/16 - Gahramanli v. Azerbaijan; 15076/17 - Somogyi v. Hungary; 34161/19 - Gültekin c. Turquie; 38979/19 - Sama c. Turquie; 42048/19 - Şorli c. Turquie; 23314/19 - Üçdağ c. Turquie; 41482/19 - Özdemir c. Turquie; 57344/19 - Akbaba c. Turquie. S. zur besonderen Frage verbotener politischer Werbung eines Kandidaten über Facebook am Wahltag 59794/18 - Năstase v. Republic of Moldova; s. auch betr. VKontakte: 8921/15, 10964/15, 14673/17 und 30328/17 - Garapova, Aronson, Zavolgin und Bondar v. Russia; 10271/12 - Kilin v. Russia; 14856/16 - Podchasov v. Russia; 17942/17 - Luzgin v. Russia; 25309/15 - Byvshev v. Russia; 33527/16 - Titarenko v. Russia; 3973/16 - Kashapov v. Russia; 17548/15 und 67686/16 - Polyudova v. Russia; 83310/17 - Romanova v. Russia; 13550/16 - Zharinov v. Russia; 2171/18 - Kochkin v. Russia; 618/18 - Sokoloskiy v. Russia.

202 S. die anhängigen Rechtssachen: 35786/19 - Melike c. Turquie; 41482/19 - Özdemir c. Turquie.

203 S. die anhängigen Rechtssachen: 56317/16 u.a. - Kasimov u.a. v. Russia (betr. alle Verhaltensweisen in den genannten Netzwerken). S. auch speziell zu Aktivitäten auf YouTube die anhängigen Rechtssachen: 51127/18 - Stefanov v. Bulgaria; 618/18 - Sokoloskiy v. Russia.

204 EGMR, 19.7.2011 - 23954/10, Rn. 20 - Uj v. Hungary; 24.3.2020 - 33938/08, Rn. 89 - Andrushchenko v. Russia. Vgl. bereits EGMR (GK), 8.7.1999 - 23168/94, Rn. 49 - Karataş v. Turkey.

205 S. nur EGMR, 13.1.2009 - 39656/03, Rn. 29 f. - Ayhan Erdoğan v. Turkey; 28.6.2016 51000/11, Rn. 62 - Radobuljac v. Croatia = NJOZ 2018, 504.

206 EGMR, 2.2.2016 - 22947/13, Rn. 77 - Magyar Tartalomszolgáltatók Egyesülete and Index.hu Zrt v. Hungary = NJW 2017, 2091; s. auch EGMR, 19.9.2017 - 3877/14, Rn. 80 f. - Tamiz v. UK (auch zur Ernsthaftigkeit von Online-Kommentaren). 
zu begrüßen. ${ }^{207}$ Es bedarf indes einer genauen Beobachtung der weiteren Entwicklung dieser Rechtsprechungslinie. Die bisherigen Aussagen eröffnen Raum für Argumentationen, die einer wahrgenommenen Verrohung der Art und Weise des öffentlichen Diskurses ${ }^{208} \mathrm{zu}$ allgemeiner grundrechtlicher Akzeptanz verhelfen könnten. Die noch ausstehende Entscheidung in der Rechtssache „Buda v. Poland“209 bietet allen Anlass dafür, in diesem Bereich für klarere Verhältnisse zu sorgen. Im Ausgangsfall lehnte das polnische Berufungsgericht die Verletzung der Persönlichkeitsrechte des Beschwerdeführers u.a. mit der Begründung ab, dass zum einen regelmäßige Teilnehmer an Diskussionen in öffentlichen Foren als Personen des öffentlichen Lebens (,public figures“) zu erachten seien und entsprechend nur einen abgeschwächten Schutzanspruch geltend machen könnten, ${ }^{210}$ sowie zum anderen dass die Sprache im Internet zwar ungehobelt, barsch und von gesellschaftlich anerkannten Standards abweichend (,blunt, curt and different from socially accepted standards"), aber dort auch akzeptiert und üblich sein möge. Letztgenannter Befund stimmt insbesondere angesichts der im Streit stehenden Äußerungen bedenklich. Dabei wird auch von Interesse sein, wie der EGMR diese Rechtsprechungslinie zu seiner gefestigten Judikatur betreffend „Hate Speech“ $^{\text {“211 }}$ - unter besonderer Berücksichtigung von Art. 17 EMRK ${ }^{212}$ - ausrichtet. Bei Anwendung der EMRK als ,living instrument“ sollte der EGMR zwar auf einen Wandel des Kommunikationsgebarens reagieren, dabei jedoch auch mögliche Auswirkungen auf Betroffene und die Debattenkultur im Internet ${ }^{213}$ berücksichtigen.

\section{Fazit}

Die vorangegangene Bestandsaufnahme hat gezeigt, dass der EGMR die Befürchtung, das Netz werde womöglich nie derselben Regulierung und Kontrolle unterliegen wie die Printmedien, ${ }^{214}$ hinter sich gelassen hat. In den vergangenen Jahren hat der Gerichtshof die Herausforderung angenommen, seinen Beitrag zu dem komplexen Unterfangen der Präzisie-

207 S. zur besonderen Berücksichtigung von ,low level digital speech“ bereits Rowbottom, CLJ 2012, 355 ( $370 \mathrm{ff}$.).

208 S. dazu m. w. Nw. Kümpel/Rieger, Wandel der Sprach- und Debattenkultur in sozialen OnlineMedien, 2019, S. $13 \mathrm{ff}$.

209 38940/13 - Buda v. Poland, betr. offensive und vulgäre Kommentare in Internetdiskussionen.

210 Ausgehend von EGMR, 8.7.1986 - 9815/82, Rn. 42 - Lingens v. Austria = NJW 1987, 2143; jüngst EGMR, 8.1.2019 - 47881/11, Rn. 30 - Prunea v. Romania.

211 S. dazu Press Unit des EGMR, Fact Sheet zu „Hate Speech“, März 2020.

212 S. dazu aufschlussreich Jurisconsult, Guide on Article 17 of the European Convention on Human Rights, 31.8.2019.

213 S. dazu Geschke/Klaßen/Quent/Richter, \#Hass im Netz: Der schleichende Angriff auf unsere Demokratie, 2019, S. 27 ff.; Kümpel/Rieger, Wandel der Sprach- und Debattenkultur in sozialen Online-Medien, 2019, S. 19 ff. m. zahlr. Nw.

214 S. zur anfänglichen Skepsis des Gerichtshofs EGMR, 5.5.2011 - 33014/05, Rn. 63 - Editorial Board of Pravoye and Shtekel v. Ukraine; 16.7.2013 - 33846/07, Rn. 58 - Węgrzynowski and Smolczewski v. Poland $=$ AfP 2014, 517. 
rung eines - zumindest regionalen - Regulierungsrahmens für das World Wide Web zu leisten. Systembedingt hat die internetbezogene Fortentwicklung seiner Rechtsprechung zu Art. 10 EMRK erst in jüngerer Zeit an Momentum gewonnen. Die Wellen von Diskussionen, die auf der Ebene der Konventionsstaaten schon länger toben, haben den EGMR erst mit einiger Verzögerung erreicht. Die diversen anhängigen Verfahren lassen jedoch erahnen, dass die Impulse aus Straßburg in Zukunft nicht nur zahlreicher, sondern auch stärker werden dürften. Inwieweit der aktuell vorgefundene „Flickenteppich“ an Entscheidungen in diesem hochdynamischen Bereich - auch im europäischen Grundrechtsverbund - noch zu einer konsistenten Internet-Judikatur verwoben wird, bleibt mit Spannung zu erwarten und mit dogmatischem Interesse kritisch zu begleiten. Umso mehr erscheint es treffend, diesen Beitrag nicht einfach zu beenden, sondern mit folgenden Worten zu beschließen: to be continued ...

\section{Literaturverzeichnis}

Adelberg, P. N., Rechtspflichten und -grenzen der Betreiber sozialer Netzwerke - Zum Umgang mit nutzergenerierten Inhalten, 2020 (Berlin: Springer Fachmedien).

Aguerre, C., Right of access to the internet - global approaches. In M. Susi (Ed.), Human Rights, Digital Society and the Law - A Research Companion, 2019 (Abingdon \& New York: Routledge), S. 31-43.

Angelopoulos, C., MTE v Hungary: A new ECtHR judgment on intermediary liability and freedom of expression, Journal of Intellectual Property Law \& Practice (JIPLP), Vol. 11 (2016) Nr. 8, S. $582-584$.

dies. \& Smet, S., Notice-and-fair-balance: how to reach a compromise between fundamental rights in European intermediary liability, Journal of Media Law (JML), Vol. 8 (2016) Nr. 2, S. 266-301.

Article 19, The Right to Blog, 2013 (London: Article 19) (abrufbar unter: https://www.article19.org/wp -content/uploads/2018/02/Right-to-Blog-EN-WEB.pdf).

Barata Mir, J. \& Bassini, M., Freedom of expression in the Internet: trends of the case law of the European Court of Human Rights. In O. Pollicino \& G. Romeo (Eds.), The Internet and Constitutional Law: The protection of fundamental rights and constitutional adjudication in Europe, 2016 (Abingdon \& New York: Routledge), S. 71-93.

Barnitzke, B., Link auf rechtswidrige Kopiersoftware in Pressebericht zulässig - „AnyDVD“ - Kommentar, Kommunikation und Recht (K\&R), Vol. 14 (2011) Nr. 5, S. 329-331.

Becker, C., Das Recht auf Vergessenwerden, 2019 (Tübingen: Mohr Siebeck).

Benedek, W./Kettemann, M. C., Freedom of expression and the Internet, 2013 (Strasbourg: Council of Europe) (abrufbar unter: https://rm.coe.int/prems-167417-gbr-1201-freedom-of-expression-on-inte rnet-web-16x24/1680984eae).

Bode, L., Anspruch auf Internet im Gefängnis? - Zugleich eine Besprechung von EGMR, Urt. v. 17.1.2017 - 21575/08, Zeitschrift für Internationale Strafrechtsdogmatik (ZIS), Vol. 12 (2017) Nr. 6, S. 348-353.

ders., Im toten Winkel - Internetnutzung durch Strafgefangene, Forum Strafvollzug (FS), Vol. 67 (2018) Nr. 3, S. 219-222.

ders., Der Einsatz internetbasierter Medien im Strafvollzug, Neue Kriminalpolitik (NK), Vol. 31 (2019) Nr. 1, S. 30-45.

ders., Kein Zugang zu Computern für Strafgefangene - Anmerkung, Neue Zeitschrift für Strafrecht Rechtsprechungs-Report (NStZ-RR), Vol. 24 (2019) Nr. 6, S. 192. 
ders., Zugang eines Sicherungsverwahrten zum Internet zu Weiterbildungszwecken - Anmerkung, Neue Zeitschrift für Strafrecht - Rechtsprechungs-Report (NStZ-RR), Vol. 24 (2019) Nr.9, S. 295-296.

Brings-Wiesen, T., Das Phänomen der „Online Hate Speech“ aus juristischer Perspektive. In K. Kaspar, L. Gräßer \& A. Riffi (Hrsg.), Online Hate Speech - Perspektiven auf eine neue Form des Hasses, 2017 (Düsseldorf \& München: kopaed), S. 35-48.

Brost, L., Das Internet muss vergessen - Veränderte Kriterien zu sog. Online-Archiven in Zeiten von Google \& Co., AfP - Zeitschrift für Medien- und Kommunikationsrecht (AfP), Vol. 46 (2015) Nr. 5, S. 407-409.

Brunner, L., The Liability of an Online Intermediary for Third Party Content - The Watchdog Becomes the Monitor: Intermediary Liability after Delfi v Estonia, Human Rights Law Review (HRLR), Vol. 16 (2016) Nr. 1, S. 163-174.

Buffa, F., Freedom of Expression in the Internet Society, 2016 (Milano: Key).

Cole, M. D., Etteldorf, C. \& Ullrich, C., Cross-Border Dissemination of Online Content - Current and Possible Future Regulation of the Online Environment with a Focus on the EU E-Commerce-Directive, 2020 (Baden-Baden: Nomos).

Dörr, D., Holznagel, B. \& Picot, A., Legitimation und Auftrag des öffentlich-rechtlichen Fernsehens in Zeiten der Cloud, Zeitschrift für Urheber- und Medienrecht (ZUM), Vol. 60 (2016) Nr. 11, S. 920-947.

Esser, R., Internet für Strafgefangene - Neue Impulse durch den EGMR, Neue Zeitschrift für Strafrecht (NStZ), Vol. 38 (2018) Nr. 3, S. 121-127.

ders., Internetzugang in der Sicherungsverwahrung - Praxiskommentar, Neue Zeitschrift für Strafrecht (NStZ), Vol. 40 (2020) Nr. 2, S. 107-109.

Fathaigh, R. Ó., The chilling effect of liability for online reader comments, European Human Rights Law Review (EHRLR), Nr. 4/2017, S. 387-393.

Gomille, C., Aktuelles zum datenschutzrechtlichen Auslistungsanspruch - Zugleich Anmerkung zu EuGH, Urteile vom 24.9.2019 - C-136/17 und C-507/17 (ZUM 2019, 813 - GC u.a./CNIL und ZUM 2019, 824 - Google/CNIL), Zeitschrift für Urheber- und Medienrecht (ZUM), Vol. 64 (2020) Nr. 2, S. 123-128.

Grabenwarter, C. (Hrsg.), Europäischer Grundrechtsschutz, Bd. 2 der Enzyklopädie Europarecht (hrsgg. von A. Hatje \& P.-C. Müller-Graff), 2014 (Baden-Baden: Nomos).

Grages, J.-M. \& Neben, G., Medienfreiheit vs. Datenschutzgarantien - Zugleich Kommentar zu EuGH, Urteil vom 14.2.2019 - C-345/17, K\&R 2019, 252 ff., Kommunikation und Recht (K\&R), Vol. 22 (2019) Nr. 5, S. 300-303.

Feldmann, T., Pflicht zur Suchmaschinenpessimierung qua Störerhaftung? - Zugleich Kommentar zu OLG Hamburg, Urteil vom 7.7.2015 - 7 U 29/12, K\&R 2015, 668 ff., Kommunikation und Recht (K\&R), Vol. 18 (2015) Nr. 10, S. 634-637.

Geschke, D., Klaßen, A., Quent, M. \& Richter, C., \#Hass im Netz: Der schleichende Angriff auf unsere Demokratie - Eine bundesweite repräsentative Untersuchung, 2019 (Berlin: Amadeu Antonio Stiftung) (abrufbar unter: https://www.demokratie-leben.de/zusatzseiten/forschungsbericht-zu-hass-im -netz.html).

Haug, T., Die Haftung von Internetportalen für verletzende Nutzerkommentare - Zugleich Anmerkung zu EGMR, Urt. v. 10.10.2013 - 64569/09 - Delfi/Estland, AfP 2014, 46, AfP - Zeitschrift für Medien- und Kommunikationsrecht (AfP), Vol. 45 (2014) Nr. 1, S. 27-30.

ders., Die Zulässigkeit von Altmeldungen in Online-Archiven - Zugleich Anmerkung zu EGMR v. 16.7.2013 - 33846/07, AfP - Zeitschrift für Medien- und Kommunikationsrecht (AfP), Vol. 45 (2014) Nr. 6, S. 503-505.

ders., Die Pflicht deutscher Gerichte zur Berücksichtigung der Rechtsprechung des EGMR, Neue Juristische Wochenschrift (NJW), Vol. 71 (2018) Nr. 37, S. 2674-2677. 
Heckmann, D. (Hrsg.), juris PraxisKommentar Internetrecht, Online-Kommentar, 6. Aufl. 2019 (Saarbrücken: Juris).

Heldt, A. P., Pflicht zu weltweiter Löschung: konsequente oder ausufernde Auslegung? - Anmerkung zum Urteil des EuGH v. 3.10.2019, Rs. C-18/18 (Glawischnig-Piesczek), Europarecht (EuR), Vol. 55 (2020) Nr. 2, S. 238-246.

dies., Loi Avia: Frankreichs Verfassungsrat kippt Gesetz gegen Hass im Netz, JuWissBlog Nr. 96/2020 v. 23.6.2020 (abrufbar unter: https://www.juwiss.de/96-2020/).

Heißl, G., EGMR und Internet - Ein Überblick über die Rechtsprechung. In J. Taeger (Hrsg.), IT und Internet - mit Recht gestalten, Deutsche Stiftung für Recht und Informatik Tagungsband (DSRITB) Herbstakademie, 2012 (Oldenburg: OlWIR), S. 61-80.

Höch, D., Der Weg ist richtig, die Straße noch im Bau - Zugleich Kommentar zu OLG Hamburg, Urteil vom 7.7.2015 - 7 U 29/12, K\&R 2015, 668 ff., Kommunikation und Recht (K\&R), Vol. 18 (2015) Nr. 10, S. 632-634.

Holoubek, M., Medienfreiheit in der Europäischen Menschenrechtskonvention, AfP - Zeitschrift für Medien- und Kommunikationsrecht (AfP), Vol. 34 (2003) Nr. 3, S. 193-201

Holznagel, D., Put-back-Ansprüche gegen soziale Netzwerke: Quo Vadis? - Bestandsaufnahme nach BVerfG v. 22.5.2019 zu "Der III. Weg" (1 BvQ 42/19) und vor dem Hintergrund aktueller rechtspolitischer Forderungen, Computer und Recht (CR), Vol. 35 (2019) Nr. 8, S. 518-526.

ders., Verpflichtung von Host-Providern zum Auffinden und Löschen wort- und sinngleicher Verletzungen - Anmerkung zu EuGH, Urteil vom 3.10.2019 - C-18/18 - Glawischnig-Piesczek/Facebook Ireland (ZUM 2019, 905), Zeitschrift für Urheber- und Medienrecht (ZUM), Vol. 63 (2019) Nr. 12, S. 910-913.

Hovlid, E., Finding a judicial definition of journalism - A challenging exercise in the digital age. In M. Susi (Ed.), Human Rights, Digital Society and the Law - A Research Companion, 2019 (Abingdon \& New York: Routledge), S. 209-224.

Husovec, M., General monitoring of third-party content: compatible with freedom of expression?, Journal of Intellectual Property Law \& Practice (JIPLP), Vol. 11 (2016) Nr. 1, S. 17-20.

Jurisconsult, Guide on Article 17 of the European Convention on Human Rights, 31.8.2019 (abrufbar unter: https://www.echr.coe.int/Documents/Guide_Art_17_ENG.pdf).

Kahl, J. \& Piltz, C., Wer hat Vorfahrt: Datenschutz oder Meinungs- und Pressefreiheit? - Zu den Abweichungsbefugnissen der Mitgliedstaaten nach der DSGVO und dem gesetzgeberischen Anpassungsbedarf im nationalen Recht, Kommunikation und Recht (K\&R), Vol. 21 (2018) Nr. 5, S. 289-295.

Karpenstein, U. \& Mayer, F. C. (Hrsg.), Konvention zum Schutz der Menschenrechte und Grundfreiheiten, 2. Aufl. 2015 (München: Beck).

Keller, D., Facebook Filters, Fundamental Rights, and the CJEU's Glawischnig-Pieszek Ruling, GRUR International - Journal of European and International IP Law (GRUR Int.), Vol. 69 (2020) Nr. 5, S. 616-623.

Kersten, J., Anonymität in der liberalen Demokratie, Juristische Schulung (JuS), Vol. 57 (2017) Nr. 3, S. 193-203.

Kettemann, M. C. \& Benedek, W., Freedom of Expression Online. In M. Susi (Ed.), Human Rights, Digital Society and the Law - A Research Companion, 2019 (Abingdon \& New York: Routledge), S. 58-74.

Klass, $N$., Das Recht auf Vergessen(-werden) und die Zeitlichkeit der Freiheit - Zugleich Besprechung der Beschlüsse des BVerfG vom 6.11.2019 - 1 BvR 16/13 - Recht auf Vergessen I (ZUM 2020, 58) sowie 1 BvR 276/17 - Recht auf Vergessen II (ZUM-RD 2020, 1), Zeitschrift für Urheber- und Medienrecht (ZUM), Vol. 64 (2020) Nr. 4, S. 265-278. 
Knauer, F., Die Rechtsprechung des EGMR zur Nutzung des Internets im Strafvollzug - Eine Besprechung der Entscheidungen Kalda gegen Estland und Jankowskis gegen Litauen. In H. Pollähne \& C. Lange-Joest (Hrsg.), www.Wahnsinn-Wohl-Wehe.de? - Gefangen(e) im Netz zwischen cyberForensik und Kriminalpolitik 2.0, 2018 (Berlin: LIT), S. 43-56.

Koltay, A., New Media and Freedom of Expression - Rethinking the Constitutional Foundations of the Public Sphere, 2019 (Oxford: Hart).

Koreng, A., Das „Recht auf Vergessen“ und die Haftung von Online-Archiven - Schlussfolgerungen für Pressearchive aus der EuGH-Entscheidung „Google Spain“, AfP - Zeitschrift für Medien- und Kommunikationsrecht (AfP), Vol. 46 (2015) Nr. 6, S. 514-518

Kovacs, A., Die Haftung der Host-Provider für persönlichkeitsrechtsverletzende Internetäußerungen, 2018 (Baden-Baden: Nomos).

Kröner, L., Online-Archive im Lichte der EMRK - Zugleich Kommentar zu EGMR, Entscheidung vom 28.6.2018 - 60798/10 und 65599/10, Kommunikation und Recht (K\&R), Vol. 21 (2018) Nr. 9, S. 544-548.

Kümpel, A. S. \& Rieger, D., Wandel der Sprach- und Debattenkultur in sozialen Online-Medien - Ein Literaturüberblick zu Ursachen und Wirkungen von inziviler Kommunikation, 2019 (Berlin: Konrad-Adenauer-Stiftung) (abrufbar unter: https://www.kas.de/de/einzeltitel/-/content/wandel-der-spr ach-und-debattenkultur-in-sozialen-online-medien).

Lepsius, O., Kontextualisierung als Aufgabe der Rechtswissenschaft, JuristenZeitung (JZ), Vol. 74 (2019) Nr. 17, S. 793-802.

Letsas, G., The ECHR as a living instrument: its meaning and legitimacy. In A. Føllesdal, B. Peters \& G. Ulfstein (Eds.), Constituting Europe - The European Court of Human Rights in a National, European and Global Context, 2013 (Cambridge: Cambridge University Press), S. 106-141.

Mackinnon, R., Hickock, E., Bar, A. \& Lim, H., Fostering Freedom Online: The Role of Internet Intermediaries, 2014 (Paris \& Reston: UNESCO \& Internet Society) (abrufbar unter: https://unesdoc.un esco.org/ark:/48223/pf0000231162).

MacMillan, I., Enforcement Through the Network: The Network Enforcement Act and Article 10 of the European Convention on Human Rights, Chicago Journal of International Law (CJIL), Vol. 20 (2019) Nr. 1, S. 252-290.

Mann, R., Die Rzeczpospolita-Entscheidung des EGMR und ihre Folgen - Online-Archive in der Rechtsprechung des EGMR - Zugleich Kommentar zu EGMR, Entscheidung vom 16.7.2013 33846/07, K\&R 2013, 570, Kommunikation und Recht (K\&R), Vol. 16 (2013) Nr. 9, S. 553-557.

ders., Kommentar zu Keine Persönlichkeitsrechtsverletzung durch Namensnennung im Online-Archiv - BVerfG, Beschluss vom 25. 2. 2020, - 1 BvR 1282/17, Kommunikation und Recht (K\&R), Vol. 23 (2020) Nr. 6, S. 434-435.

McGonagle, T., The Council of Europe and Internet Intermediaries: A Case Study of Tentative Posturing. In R. F. Jørgensen (Ed.), Human Rights in the Age of Platforms, 2019 (Cambridge: MIT Press), S. 227-254.

Meyer, S. \& Stakowski, M., Muss Google Suchergebnisse redaktionell sortieren? - Zugleich Kommentar zu EuGH, Urteil vom 24. 9. 2019 - C-136/17, K\&R 2019, 710 ff., Kommunikation und Recht (K\&R), Vol. 22 (2019) Nr. 11, S. 677-680.

Michel, S., Datenschutzrechtliche Anforderungen an Bloggerinnen und Bürgerjournalistinnen Braucht das Verhältnis von Presserecht und Datenschutz ein Update? In S. Hetmank \& C. Rechenberg (Hrsg.), Kommunikation, Kreation und Innovation - Recht im Umbruch?, 2019 (Baden-Baden: Nomos), S. 131-156.

ders., Reformbedarf des datenschutzrechtlichen Medienprivilegs für Bloggerinnen?, AfP - Zeitschrift für das gesamte Medienrecht (AfP), Vol. 49 (2019) Nr. 6, S. 490-495.

Milker, J., Die Umsetzung des „Rechts auf Vergessenwerden“ im deutschen Recht - Der Datenschutz als Taktgeber für das Äußerungsrecht, 2019 (Wiesbaden: Springer Fachmedien). 
Milstein, A., EGMR: Haftung eines Forenbetreibers für anonyme Nutzerkommentare mit Meinungsfreiheit vereinbar - Anmerkung, Multimedia und Recht (MMR), Vol. 17 (2014) Nr. 1, S. 41-42.

Oermann, M., Gewährleistung der Möglichkeit internetbasierter Kommunikation - Eine Vermessung des grundgesetzlichen Schutzkonzepts, 2018 (Baden-Baden: Nomos).

O'Leary, S., Balancing rights in a digital age, Irish Jurist, Vol. 59 (2018), S. 59-92.

Oręziak, B., Judicial Dialogue between the European Court of Human Rights and the Court of Justice of the European Union in the Field of Legal Liability for Posting Hyperlinks, International Community Law Review (ICLR), Vol. 21 (2019) Nr. 5, S. 432-448.

Ory, S., Das Äußerungsrecht auf dem Zeitstrahl, Archiv (AfP), Vol. 50 (2020) Nr. 2, S. 119-125.

Oster, J., Media Freedom as a Fundamental Right, 2015 (Cambridge: Cambridge University Press).

ders., European and International Media Law, 2017 (Cambridge: Cambridge University Press).

ders., Kommunikationsdeliktsrecht - Eine transnationale Untersuchung am Beispiel des Ehrschutzes, 2019 (Tübingen: Mohr Siebeck).

Paal, B. P. \& Hennemann, M., Online-Archive im Lichte der Datenschutz-Grundverordnung, Kommunikation und Recht (K\&R), Vol. 20 (2017) Nr. 1, S. 18-23.

Paefgen, F., Der von Art. 8 EMRK gewährleistete Schutz vor staatlichen Eingriffen in die Persönlichkeitsrechte im Internet, 2017 (Heidelberg \& Berlin: Springer).

von Pentz, V., Ausgewählte Fragen des Medien- und Persönlichkeitsrechts im Lichte der aktuellen Rechtsprechung des VI. Zivilsenats, AfP - Zeitschrift für das gesamte Medienrecht (AfP), Vol. 50 (2020) Nr. 2, S. 93-109.

Petri, T., EGMR: Haftung des Providers eines Internetportals für beleidigende Kommentare - Anmerkung, Zeitschrift für Datenschutz (ZD), Vol.5 (2015) Nr. 10, S. 469-470.

Press Unit des EGMR, Fact Sheet zu „Access to Internet and freedom to receive and impart information and ideas", Juni 2020 (abrufbar unter: https://www.echr.coe.int/Documents/FS_Access_Intern et_ENG.pdf).

dies., Fact Sheet zu „New technologies“, Februar 2020 (abrufbar unter: https://www.echr.coe.int/Docu ments/FS_New_technologies_ENG.pdf).

dies., Fact Sheet zu „Hate Speech“, März 2020 (abrufbar unter: https://www.echr.coe.int/Documents/F S_Hate_speech_ENG.pdf).

Reinhardt, J., „Recht auf Vergessen“ auf Französisch, Datenschutz und Datensicherheit (DuD), Vol. 44 (2020) Nr. 6, S. 361-364.

Regenfus, T., Auswirkungen von Zeitablauf und Erkenntnisgewinn auf die Zulässigkeit der Verdachtsberichterstattung, Zeitschrift für Urheber- und Medienrecht (ZUM), Vol. 64 (2020) Nr. 4, S. 278-287.

Research Division des EGMR, Internet: case-law of the European Court of Human Rights, Juni 2015 (abrufbar unter: https://www.echr.coe.int/Documents/Research_report_internet_ENG.pdf).

Research and Library Division des EGMR, Article 10: Expression and advertising of political positions through the media/Internet in the context of elections/referendums, 22.8.2018 (abrufbar unter: https://www.echr.coe.int/Documents/Research_report_expression_advertising_media_elections_E NG.pdf).

Rombey, S., Die Geltung des Medienprivilegs für YouTuber - Neue Impulse aus Luxemburg als Chance für das neue Datenschutzrecht, Zeitschrift für Datenschutz (ZD), Vol. 9 (2019) Nr. 7, S. 301-305.

Roth, Y. \& Pickles, N., Updating our Approach to Misleading Information, Twitter-Blog v. 11.5.2020 (abrufbar unter: https://blog.twitter.com/en_us/topics/product/2020/updating-our-approach-to-misl eading-information.html).

Rowbottom, J., To Rant, Vent and Converse: Protecting Low Level Digital Speech, Cambridge Law Journal (CLJ), Vol. 71 (2012) Nr. 2, S. 355-383. 
Schapiro, L., Anhaltende Rechtsunsicherheit für die Betreiber von Internetmeinungsportalen? - Das Urteil des EGMR „Delfi AS v. Estonia“ und seine Auswirkungen auf die deutsche Rechtslage, Zeitschrift für Urheber- und Medienrecht (ZUM), Vol. 58 (2014) Nr. 3, S. 201-210.

Schierbaum, L., Sorgfaltspflichten von professionellen Journalisten und Laienjournalisten im Internet Zugleich ein Beitrag zur rechtlichen Einordnung einer neuen Publikationskultur, 2016 (Baden-Baden: Nomos).

Spano, R., Intermediary Liability for Online User Comments under the European Convention on Human Rights, Human Rights Law Review (HRLR), Vol. 17 (2017) Nr. 4, S. 665-679.

Spindler, G., Weltweite Löschungspflichten bei Persönlichkeitsverletzungen im Internet, Neue Juristische Wochenschrift (NJW), Vol. 72 (2019) Nr. 45, S. 3274-3277.

Spindler, G. \& Schuster, F. (Hrsg.), Recht der elektronischen Medien, 4. Aufl. 2019 (München: Beck).

Stern, K. \& Sachs, M. (Hrsg.), Europäische Grundrechte-Charta, 2016 (München: Beck).

Trentmann, C., Die (un)geklärte Rechtslage bei Altberichten in Online-Archiven - Kritischer Überblick zum aktuellen Entwicklungsstand der Rechtsprechung, Multimedia und Recht (MMR), Vol. 19 (2016) Nr. 11, S. 731-735.

Tully, S., A Human Right to Access the Internet? Problems and Prospects, Human Rights Law Review (HRLR), Vol. 14 (2014) Nr. 2, S. 175-195.

Uerpmann-Wittzack, R. \& Jankowska-Gilberg, M., Die Europäische Menschenrechtskonvention als Ordnungsrahmen für das Internet, Multimedia und Recht (MMR), Vol. 11 (2008) Nr. 2, S. 83-89.

Vajić, N. \& Voyatzis, P., The Internet and freedom of expression: A „brave new world“ and the European Court of Human Rights` Evolving Case Law. In J. Casadevall, E. Myjer, M. O’Boyle \& A. Austin (Eds.), Freedom of Expression: Essays in honour of Nicolas Bratza, 2012 (Oisterwijk: Wolf), S. 391-420.

Verweyen, U. \& Ruf, I., OLG Hamburg: Löschung alter Beiträge aus Online-Archiv - Anmerkung, Multimedia und Recht (MMR), Vol. 18 (2015) Nr. 11, S. 772-774.

Volkmann, C., Verantwortlichkeit von Plattformbetreibern für rechtsverletzende Nutzerkommentare Zugleich Kommentar zu EGMR, 10.10.2013, Application No. 64569/09 - Delfi AS v. Estonia, Kommunikation und Recht (K\&R), Vol. 16 (2013) Nr. 12, S. 762-764.

Voorhoof, D., Same standards, different tools? The ECtHR and the protection and limitations of freedom of expression in the digital environment. In M. O'Boyle (Ed.), Human rights challenges in the digital age: Judicial perspectives, 2020 (Strasbourg: Council of Europe), S. 11-46.

ders., van Loon, A., Vier, C. \& Fathaigh, R. Ó. and McGonagle, Tarlach (Ed. Sup.), Freedom of Expression, the Media and Journalists: Case-law of the European Court of Human Rights, IRIS Themes Vol. III, 5th Ed. 2020 (Strasbourg: European Audiovisual Observatory) (abrufbar unter: https://rm.coe.int/iris-themes-vol-iii-ed-2020-en-2/16809e45e7).

Voßkuhle, A., Rechtspluralismus als Herausforderung - Zur Bedeutung des Völkerrechts und der Rechtsvergleichung in der Rechtsprechung des Bundesverfassungsgerichts, Zeitschrift für ausländisches öffentliches Recht und Völkerrecht (ZaöRV), Vol. 79 (2019) Nr. 3, S. 481-501.

Wagner, G., Haftung von Plattformen für Rechtsverletzungen - Teil 1: Gewerblicher Rechtsschutz und Urheberrecht (GRUR), Vol. 122 (2020) Nr. 4, S. 329-338; Teil 2: Nr. 5, S. 447-457.

Weberling, J. \& Bergann, J., Aktuelle Fragen der Umsetzung des Medienprivilegs der DSGVO, AfP Zeitschrift für das gesamte Medienrecht (AfP), Vol. 49 (2019) Nr. 4, S. 293-298.

Wein, M., Free Flow of Information, Archiv für Urheber-, Film-, Funk- und Theaterrecht (UFITA), Vol. 105 (1987), S. 137-161.

Ziegelmayer, D., Vorsichtsmaßnahmen statt Vorab-Kontrollen: Zur Haftung für „Hass Postings“ - Zugleich Kommentar zu EGMR, 2.2.2016 - Application No. 22947/13, Kommunikation und Recht (K\&R), Vol. 19 (2016) Nr. 4, S. 228-229.

Zipursky, R., Nuts About NETZ: The Network Enforcement Act and Freedom of Expression, Fordham International Law Journal, Vol. 42 (2019) Nr. 4, S. 1325-1374. 